\title{
Does particle creation mechanism favour formation of black hole or naked singularity?
}

\author{
Sudipto Bhattacharjee ${ }^{1, \mathrm{a}}$, Subhajit Saha ${ }^{2, \mathrm{~b}}$, Subenoy Chakraborty ${ }^{1, \mathrm{c}}$ \\ ${ }^{1}$ Department of Mathematics, Jadavpur University, Kolkata, West Bengal 700032, India \\ ${ }^{2}$ Department of Mathematics, Panihati Mahavidyalaya, Sodepur, West Bengal 700110, India
}

Received: 2 August 2017 / Accepted: 3 June 2018 / Published online: 12 June 2018

(c) The Author(s) 2018

\begin{abstract}
The paper deals with collapse dynamics of a spherically symmetric massive star in the framework of non-equilibrium thermodynamic prescription through particle creation mechanism. The matter content in the star is in the form of perfect fluid with barotropic equation of state, and the dissipative phenomena due to non-equilibrium thermodynamics is in the form of bulk viscosity. For simplicity, the thermodynamic system is chosen to be adiabatic so that the effective bulk viscous pressure is linearly related to the particle creation rate. As a result, the evolution of the collapsing star also depends on the particle creation rate. By proper choice of creation rate as a function of the Hubble parameter, it is found that the end state of the collapse may be either a black hole $(\mathrm{BH})$ or a naked singularity (NS).
\end{abstract}

\section{Introduction}

It is found from Supernovae Type Ia (SNIa) data [1-4] that our universe is going through an accelerated phase of expansion. It is also indirectly supported by various indirect observations like temperature anisotropies of CMB [5], Baryon Acoustic Oscillations (BAO) [6], Weak Lensing (WL) [7], Integrated Sachs-Wolfe effect (ISW) [8] etc. Gravitational collapse in the expanding universe is studied in literature [9]. Also, gravitational particle creation in expanding universe studied in literature [10]. So, it may be interesting to study the effect of particle creation in the context of astrophysical collapse.

The study of gravitational collapse is an important issue in classical general relativity. Usually, the stellar objects such as white dwarfs and neutron stars are formed through a collapsing process. Also in astrophysical collapse one should

\footnotetext{
${ }^{a}$ e-mail: slg00sudipto@gmail.com

be-mail: subhajit1729@gmail.com

c e-mail: schakraborty.math@gmail.com
}

match the interior and exterior spacetime of the collapsing object through the proper junction conditions.

The evolution of a star is an important astrophysical phenomenon. The equilibrium configuration of a star during its thermonuclear burning is the balance of the gravitational attraction with the outward internal pressure. Subsequently, after the nuclear burning is completed the star may have again equilibrium configuration as white dwarf or neutron star depending on the mass of the star. If $M$ (mass of the star) $<1.4 M_{\odot}$ (Chandrasekhar limit), then electron degeneracy pressure will balance gravitational attraction and the star becomes a white dwarf [11] while if $1.4 M_{\odot}<M<2.5 M_{\odot}$, then neutron degeneracy pressure will balance the gravitational attraction and the star becomes a neutron star [12]. However, for more massive stars (after nuclear burning) the gravitational attraction can not be balanced by any outward pressure and the star crunches to a singularity (by singularity theorem of Penrose and Hawking [13]). On the other hand, according to the Cosmic Censorship Conjecture (CCC), proposed by Penrose [14] spacetime singularity evolved from gravitational collapse must be hidden behind the horizon i.e. $\mathrm{BH}$ is the only possible end state of collapse. Further, the CCC has no formal mathematical proof in one hand while several models related to the gravitational collapse of matter so far has been constructed where one encounters a naked singularity. Thus astrophysically, the end state of collapse of a massive star is a challenging issue today.

Long back in 1939 Oppenheimer and Snyder [15] initiated the study of gravitational collapse with interior spacetime represented by Friedmann like dust solution with a static Schwarzschild exterior. Subsequently several authors have extended this study of gravitational collapse. In the following, we shall mention some of the important and realistic generalization of the above pioneering work: (1) Misner and Sharp [16] considered the perfect fluid collapse with the same static exterior, (2) using Vaidya's [17] idea of outgoing radiation of the collapsing body, Santos and collaborators [18-21] 
considered dissipative collapsing matter by allowing radial heat flow (i.e. radiating collapse). On the other hand, Cissoko et al. [22] and Goncalves [23] studied junction conditions of a non-static collapsing object with a static interior. Gravitational collapse in the presence of dark energy has been investigated in [24] and [25].

The discovery of Hawking radiation has shown a nice interrelationship between $\mathrm{BH}$ and thermodynamics. Subsequently, it is found that there is a deep inner relationship between gravity and thermodynamics. So, it is interesting to consider the thermodynamic analysis of a collapsing massive star, which sinks under the attraction of its own gravity, and at the end of its life cycle either BH will form, or it will appear as a NS, depending on the nature of the initial data. In particular, it is curious to know the validity of the thermodynamic laws for the collapsing astrophysical object.

In recent years, a lot of works [26-32] have been done in cosmology in the perspective of non-equilibrium thermodynamics within the framework of particle creation mechanism. The main motivation of these works is to explain the well known observational evidence that our universe is going through an accelerating phase since recent past. It is found $[27,29]$ that by proper choice of the particle creation rate (as function of Hubble parameter) the late time accelerated expansion can be described in the context of Einstein's general relativistic theory (GRT) without introduction of any exotic matter (dark energy). Also recently [26] it is shown that the above models not only describe the late phase of the evolution but also describe the entire cosmic evolution since inflation to $\triangle \mathrm{CDM}$ model.

The present work is also related to the particle creation mechanism but in the context of well known astrophysical problem namely the final fate of a massive collapsing star. We shall address the question whether the particle creation mechanism favours formation of $\mathrm{BH}$ or helps the collapsing star to become a NS.

Usually, physical laws breakdown as one approaches to a spacetime singularity (where density, curvature and other physical quantities diverge), so such regions are not considered in the spacetime manifold. Hence it is speculated [50] that the thermodynamic properties on the manifold may not be smooth (i.e. regular) in the limit of approaching the singularity. As a result, the CCC [14] may be assumed to be related to the thermodynamic nature of the spacetime manifold near NS [50]. Further in the context of dynamical BHs, Hayward [51-53] introduced the notion of trapping horizon and the idea of unified first law. Subsequently, this generalization has been extended to universal thermodynamics with FRW cosmological model and it is found that there is nice equivalence between gravity and thermodynamics at cosmological scenario $[54,55]$. Due to equivalence of trapping horizon and apparent horizon for FRW model, it is sufficient to consider thermodynamic behavior at the apparent horizon (formed inside the collapsing sphere) in the limit of approach to the singularity.

The plan of the paper is as follows: In Sect. 2, the basic idea of collapsing mechanism has been presented, collapsing solutions and relevant physical properties for various choices of the particle creation rate has been shown in Sect. 3. Section 4 deals with junction conditions and relevant physical interpretations with Schwarzschild-de Sitter as the exterior spacetime. The thermodynamics of the collapsing star has been discussed in Sect. 5. A field theoretic description has been shown in Sect. 6. The paper ends with a brief discussion and concluding remarks in Sect. 7.

\section{The basic idea of collapsing mechanism}

In the present work, the matter of the collapsing star is chosen in the form of perfect fluid with barotropic equation of state $p=(\gamma-1) \rho$, while the dissipative phenomena due to nonequilibrium thermodynamics is in the form of bulk viscosity. As an astrophysical object is an element within the universe, so the non equilibrium thermodynamics of the universe will have its imprint on an astrophysical object. For simplicity, the thermodynamic system is chosen as adiabatic in nature so that the entropy per particle is chosen as constant. As a result, the effective bulk viscous pressure is determined by the particle creation rate $[26-29,33]$ as

$\Pi=-\frac{\Gamma}{3 H}(p+\rho)$,

where $\Gamma$ is the particle creation rate, $\Pi$ is the effective bulk viscous pressure (due to dissipation), and $H=\frac{\dot{a}}{a}$ is the Hubble parameter (an overdot stands for differentiation with respect to cosmic time $t$ ). It is to be noted that the particles created inside the core of the collapsing astrophysical object should be perfect fluid particles due to adiabatic nature of the thermal process.

As the dissipative phenomena of the matter field inside the massive core is bulk viscous in nature, so the spacetime inside core should be homogeneous and isotropic. Hence the inside geometry is characterized by the flat Friedmann-RobertsonWalker (FRW) model

$d s_{-}^{2}=d t^{2}-a^{2}(t)\left(d r^{2}+r^{2} d \Omega_{2}^{2}\right)$,

and it is a particular case of the inhomogeneous Oppenheimer-Snyder model [15]. Here $a(t)$ is the scale factor, and $d \Omega_{2}^{2}=d \theta^{2}+\sin ^{2} \theta d \phi^{2}$ is the metric on unit 2-sphere. In spite of the very ideal situation of the present collapsing process it is speculated that present model captures the main features of the gravitational collapse, similar to OppenheimerSnyder model [15] that gives most of the main properties of 
a collapsing star in an otherwise flat background. Further, in analogy to cosmology where the curvature effects are not important at the early stages of the evolution [34,35], and it is assumed that the same thing happens for the late stages of the collapsing core. The main question that we shall have to address is the end state of collapse - a BH or NS, i.e. the singularity is covered by an apparent horizon or not. Further, it is to be noted that this open spacetime model will be considered as the interior region bounded by Schwarzschild-de Sitter spacetime in the following section. The boundary is described by $r=$ constant. So for the junction condition there will be no effect even if we consider the closed FRW model.

Apparent horizons are spacelike surfaces with future point converging null geodesics on both sides of the surface [36, 37]. In fact, the apparent horizon is a trapped surface lying in a boundary of a particular surface $S$. In particular, if $S$ is a two-sphere embedded in a 3D-slice $\Sigma$ of 4D spacetime $M$, and let $s^{\mu}$ be the outward-pointing spacelike unit normal to $\Sigma$ and $n^{\mu}$, the future pointing time-like unit normal to $\Sigma$, so that $k^{\mu}=s^{\mu}+n^{\mu}$ is a null vector, then the surfaces will be called marginally trapped surface if $k_{; \mu}^{\mu}=0$ holds everywhere on $S$ [37].

For the present FRW model, the apparent horizon is characterized by [38-40]

$R_{, i} R_{, j} g^{i j} \equiv(r \dot{a})^{2}-1=0$,

where $R(t, r)=r a(t)$ is the area radius and the comma in the 1.h.s. indicates partial derivatives. Here Greek indices run over 0 to 3 while Latin indices ranges over 0 and 1 with $x^{0}=t, x^{1}=r$. As the matter fields are co-moving in the spacetime described by the above metric Eq. (2) and the star is assumed to be untrapped initially, so the comoving boundary surface of the star is spacelike: $r_{\mid \Sigma}=$ constant, say $r_{\Sigma}$. Thus we have on $\Sigma$ :

$R_{, i} R_{, j} g^{i j} \equiv\left\{r_{\Sigma} \dot{a}(t)\right\}^{2}-1<0$,

i.e. $0<R_{i} H_{i}<1$, where $R_{i}, H_{i}$ are the initial area radius and Hubble parameter of the collapsing core. Here $r_{\Sigma}$ denotes the boundary of the collapsing star and we have on $\Sigma$ :

$d s_{\Sigma}^{2}=d \tau^{2}-R^{2}(\tau) d \Omega_{2}^{2}$,

where $\tau=t$ and $R(\tau)=r_{\Sigma} a(\tau)$ is the area radius of the bounding surface. The metric outside the collapsing star in general can be written in the form $[25,41]$

$d s_{+}^{2}=A^{2}(T, R) d T^{2}-B^{2}(T, R)\left(d R^{2}+R^{2} d \Omega_{2}^{2}\right)$.

In view of the exterior spacetime, the surface $\Sigma$ can be expressed as $R=R_{0}(T)$. Israel's junction conditions on the boundary have been discussed in details by Cai and Wang [25,41]. Once dependence of $A$ and $B$ on $T$ and $R$ is known, it is possible to determine the time evolution of $T, R_{0}, A$, and $B$ along the hypersurface $\Sigma$.

For gravitational collapse $\dot{a}<0$, and $R(t, r) \equiv$ $r a(t)$ denotes the geometric radius of the two spheres: $(t, r)=$ constant. The mass function due to Cahill and McVittie [42] is defined as

$m(r, t)=\frac{R}{2}\left(1+R_{, \alpha} R_{, \beta} g^{\alpha \beta}\right)=\frac{1}{2} R \dot{R}^{2}$.

Thus the total mass of the collapsing cloud is

$m(\tau)=m\left(r_{\Sigma}, \tau\right)=\frac{1}{2} R(\tau) \dot{R}^{2}(\tau)$.

Note that the inequality (4) should hold at the initial epoch so that the collapsing process starts from regular initial data. Further, if the above inequality holds throughout the collapsing process, then the collapse will evidently not form $\mathrm{BH}$.

It should be noted that although the total mass given by (5) and the global structure of the BH depends on the spacetime geometry outside the star (and also on the matching conditions) but the basic question of $\mathrm{BH}$ formation depends crucially on the development of apparent horizon inside the core - not on the matching conditions and the choice of spacetime outside the star. Although in the present work we shall address the question whether a collapsing massive star will become a BH or not at the end stages of its collapse, still we shall explicitly mention the junction conditions to compare the collapse dynamics for Schwarzschild and Schwarzschildde Sitter model as the exterior of the collapsing star.

Further, it should be mentioned that supermassive BHs (as at the galactic centre) or recently discovered quasar at redshift $z=7.085$ and mass $M=2 \times 10^{9} M_{\odot}$ [43], which is speculated to be formed from huge massive collapsing star cores of population III, has extremely large mass due to cosmological accretion mechanism and mergers in the course of their evolution. We only concentrate ourselves to the discussion related to BHs and NSs, formed from collapsing star cores.

\section{Collapsing solutions}

The basic Friedmann equations for the present model are

$3 H^{2}=8 \pi G \rho \quad$ and $\quad 2 \dot{H}=-8 \pi G(\rho+p+\Pi)$,

where the energy-momentum tensor for the matter distribution is

$T_{\mu \nu}=(\rho+p+\Pi) u_{\mu} u_{\nu}+(p+\Pi) g_{\mu \nu}$, 
having conservation equation (i.e. $T_{\nu ; \mu}^{\mu}=0$ )

$\dot{\rho}+3 H(\rho+p+\Pi)=0$.

Here $u_{\mu}$ is the unit time-like vector (normalized velocity vector) and semicolon stands for covariant differentiation. Now eliminating the dissipative effect from the Friedmann equations (6) by using the Eq. (1), the collapse dynamics is characterized by the particle creation rate as

$\frac{2 \dot{H}}{3 H^{2}}=-\gamma\left(1-\frac{\Gamma}{3 H}\right)$

where equation of state for the perfect fluid namely $p=$ $(\gamma-1) \rho$ has been used (assuming $\gamma \neq 0)$. In the present work, we shall choose $\Gamma$ as

$\Gamma=\Gamma_{3}+3 \Gamma_{0} H+\frac{\Gamma_{1}}{H}$,

with $\Gamma_{0}, \Gamma_{1}$ and $\Gamma_{3}$ as real constants and $\Gamma \neq 0$. This choice of $\Gamma$ is chosen from the recent study [29] where it describes the unified evolution of the universe from matter dominated era to late time acceleration (up to phantom barrier, asymptotically). As in course of the evolution of the universe the stars are formed in the matter dominated era so the collapsing process occurs at matter dominated era or at later stage of evolution of the universe [described by the particle creation rate (8)]. Hence the above choice of $\Gamma$ is justified in the present astrophysical context. Also in [26] it has been shown that $\Gamma \propto H$ and $\Gamma \propto \frac{1}{H}$ corresponds respectively to matter dominated and late time accelerating phase of the universe. Hence in the present study $\Gamma_{0}$ and $\Gamma_{1}$ may symbolically represent the decelerated and accelerated expansion phases respectively. Now, using Eq. (8) in (7), the evolution equation for the scale factor becomes

$\frac{\ddot{a}}{a}+\left\{\frac{3 \gamma}{2}\left(1-\Gamma_{0}\right)-1\right\} \frac{\dot{a}^{2}}{a^{2}}-\frac{\gamma \Gamma_{3}}{2} \frac{\dot{a}}{a}-\frac{\gamma \Gamma_{1}}{2}=0$,

which on integration gives

$H=\left[-H_{2}^{-1}+\mu \tanh T\right]^{-1}$

and integrating once more, we obtain

$\left(\frac{a}{a_{0}}\right)^{\mu \alpha_{1}}=e^{l T}\left[H_{2}\left\{\frac{\Gamma_{3}}{2 \Gamma_{1}} \cosh T-\mu \sinh T\right\}\right]^{m}$.

In the above solution, we have chosen $\mu^{2}=\frac{\left\{12 \Gamma_{1}\left(1-\Gamma_{0}\right)+\Gamma_{3}^{2}\right\}}{4 \Gamma_{1}^{2}}$, $\alpha_{1}=\frac{\gamma \Gamma_{1}}{2}, \quad m=\frac{\mu}{\left[\mu^{2}-\left(\frac{\Gamma_{3}}{2 \Gamma_{1}}\right)^{2}\right]}, l=\frac{H_{2}^{-1}}{\left[\mu^{2}-H_{2}^{-2}\right]}, T=\mu \alpha_{1}(t-$ $\left.t_{0}\right), H_{2}=\left(\frac{\Gamma_{3}}{2 \Gamma_{1}}\right)^{-1}$, and $a_{0}, t_{0}$ are constants of integration (with $\Gamma_{0} \neq 1$ ). The time of collapse $t_{c}$ when $a=0$ is obtained from the above Eq. (11) as

$t_{c}=t_{0}+\frac{1}{\mu \alpha_{1}}\left[\tanh ^{-1}\left(\frac{1}{H_{2} \mu}\right)\right]$.

Using this collapsing time the scale factor and Hubble parameter can respectively be written in compact form as

$$
\begin{gathered}
\left(\frac{a}{a_{0}}\right)^{\mu \alpha_{1}}=e^{l T}(\cosh T)^{m}\left[1-\frac{\tanh T}{\tanh T_{c}}\right]^{m}, \\
H=-H_{2}\left[1-\frac{\tanh T}{\tanh T_{c}}\right]^{-1},
\end{gathered}
$$

with $T_{c}=\mu \alpha_{1}\left(t_{c}-t_{0}\right)$.

The negativity of $H$ characterizes the collapsing process under consideration. If $t_{a H}$ is the time of formation of apparent horizon then from (3) the condition for appearance of apparent horizon takes the form

$R_{0} H \frac{a}{a_{0}}=-1$

i.e.

$R_{0} H_{2} e^{\left(\frac{l}{\mu \alpha_{1}}\right) T_{a H}}\left(\cosh T_{a H}\right)^{n+1}\left[1-\frac{\tanh T_{a H}}{\tanh T_{c}}\right]^{n}=1$,

with $T_{a H}=\mu \alpha_{1}\left(t_{a H}-t_{0}\right), \quad R_{0}=a_{0} r$ and $n=\frac{m}{\mu \alpha_{1}}-1$. As $\tanh x$ is an increasing function of $x$, so for real solution of the above equation for $t_{a H}$, we must have the following possibilities:

1. $t_{c}>t_{a H}$ for any real value of $n$ (except $n$ to be a positive integer).

2. $t_{c}<t_{a H}$ or $t_{c}>t_{a H}$, if $n$ is an even integer. Note that limiting situation (i.e. $t_{c}=t_{a H}$ ) is not possible for Eq. (12). Thus, depending on the value of $n$ i.e. the values of the coefficients in the expression for particle creation rate [i.e. Eq. (8)], it is possible to have either a $\mathrm{BH}$ (i.e. $\left.t_{c}>t_{a H}\right)$ or a NS (i.e. $\left.t_{c}<t_{a H}\right)$.

Therefore, the final fate of collapse of a massive star depends strongly on the choice of the particle creation rate.

We shall now discuss the collapse dynamics for the choice $\Gamma_{0}=1$. Note that this choice and the subsequent choices of $\Gamma$ are particular cases of the general choice (8) and these choices are made purely for mathematical simplicity. Thus, the evolution Eq. (9) simplifies to

$\dot{H}=\frac{\gamma}{2}\left(\Gamma_{3} H+\Gamma_{1}\right)$, 


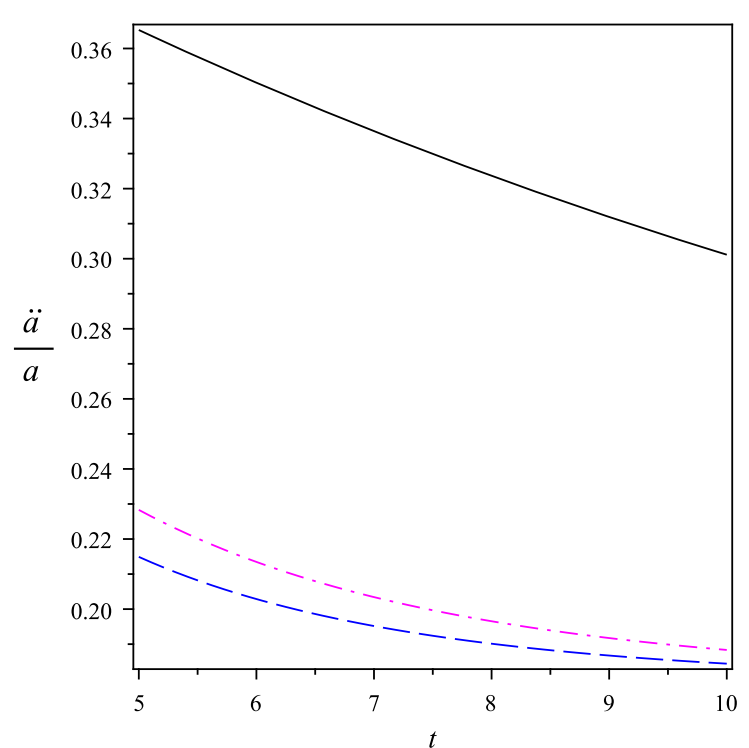

Fig. 1 The figure on the left side represents accelerating collapsing process given by first equation of (15) and the figure on the right side represents evolution of the Hubble parameter given by (10) against time $t$, respectively for $\Gamma_{0} \neq 1$. Here the dimension of $\frac{\ddot{a}}{a}$ is $s^{-2}$ and that of $t$ is $s$. In both the figures the curves in the solid line represent $\frac{\ddot{a}}{a}$ and

which has the solution

$H=-\delta+\left(H_{0}+\delta\right) e^{-\frac{\gamma \alpha}{2}\left(t-t_{0}\right)}$,

$a=a_{0} e^{-\delta\left(t-t_{0}\right)} \exp \left[-\frac{2\left(H_{0}+\delta\right)}{\gamma \alpha}\left\{e^{-\frac{\gamma \alpha}{2}\left(t-t_{0}\right)}-1\right\}\right]$,

where as before $a_{0}, H_{0}$ (which is negative) are the values of the scale factor and the Hubble parameter at $t=t_{0}$. Here, we have chosen $\alpha=-\Gamma_{3}, \mu=-\Gamma_{1}$ and $\delta=\frac{\Gamma_{1}}{\Gamma_{3}}$. From the above expression for the scale factor, we see that the present physical process, i.e. collapse of a star will take an infinite time for collapse, i.e. $t_{c}=\infty$. Using Eq. (3), the time of formation of apparent horizon is determined from the relation

$$
\begin{aligned}
& R_{0} e^{-\delta \widetilde{T}_{a H}}\left[\delta-\left(H_{0}+\delta\right) e^{-\frac{\gamma \alpha}{2} \widetilde{T}_{a H}}\right] \\
& \exp \left[-\frac{2\left(H_{0}+\delta\right)}{\gamma \alpha}\left\{e^{-\frac{\gamma \alpha}{2} \widetilde{T}_{a H}}-1\right\}\right]=1
\end{aligned}
$$

with $\widetilde{T}_{a H}=t_{a H}-t_{0}$. The above equation shows that $t_{a H}$ always has a finite solution, and hence the apparent horizon forms much earlier than the time of collapse. So the collapsing process inevitably leads to formation of a $\mathrm{BH}$. Thus the end state of collapse depends not only on the choice of the particle creation rate but also on the numerical values of the coefficients of different powers of $H$.

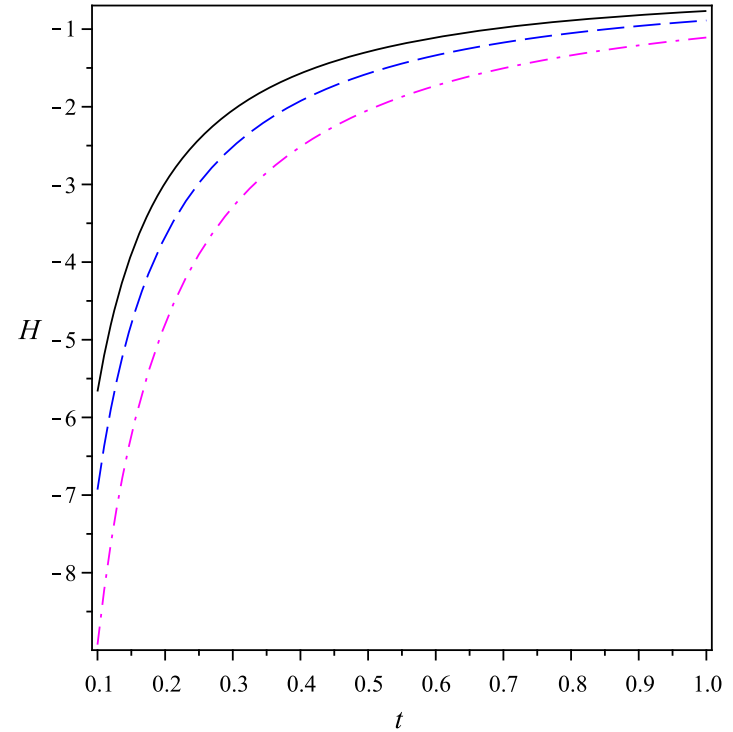

$H$, respectively for $\alpha_{1}=-0.05$ (in this case $n$ (=15) is odd). The curves in the dashed line represent $\frac{\ddot{a}}{a}$ and $H$, respectively for $\alpha_{1}=-\frac{1}{3}$ (in this case $n(=1.4)$ is a positive non integer) and the curves in the dash-dotted line represent $\frac{\ddot{a}}{a}$ and $H$, respectively for $\alpha_{1}=-\frac{4}{15}$ (in this case $n(=2)$ is even). In all the cases we have considered $\mu=\frac{\sqrt{3}}{2}$

Further, for the present collapsing process the measure of acceleration is given by

$\ddot{a} a= \begin{cases}\left\{1-\mu^{2} \alpha_{1} \operatorname{sech}^{2} T\right\} H^{2}, & \text { for } \Gamma_{0} \neq 1 \\ {\left[-\delta+\left(H_{0}+\delta\right) e^{-\frac{\gamma \alpha}{2}\left(t-t_{0}\right)}-\frac{\gamma \alpha}{4}\right]^{2}} & \\ -\left(\frac{\gamma^{2} \alpha^{2}}{16}-\frac{\gamma \mu^{2}}{2}\right), & \text { for } \Gamma_{0}=1\end{cases}$

The collapsing process (for $\Gamma_{0} \neq 1$ ) will be accelerating if $T>\cosh ^{-1}\left(\mu \sqrt{\alpha_{1}}\right)$ or $T<-\cosh ^{-1}\left(\mu \sqrt{\alpha_{1}}\right)$ (see Fig. 1) and the collapsing process will be decelerating if $-\cosh ^{-1}\left(\mu \sqrt{\alpha_{1}}\right)<T<\cosh ^{-1}\left(\mu \sqrt{\alpha_{1}}\right)$. Similarly for the choice $\Gamma_{0}=1$ (see Fig. 2), the collapsing process will be accelerating if $t>t_{0}+\frac{2}{\gamma \alpha} \ln \left(\frac{\delta+H_{0}}{\delta+\frac{\gamma \alpha}{4}-\sqrt{\frac{\gamma^{2} \alpha^{2}}{16}-\frac{\gamma \mu}{2}}}\right)$ or $t<t_{0}+\frac{2}{\gamma \alpha} \ln \left(\frac{\delta+H_{0}}{\delta+\frac{\gamma \alpha}{4}+\sqrt{\frac{\gamma^{2} \alpha^{2}}{16}-\frac{\gamma \mu}{2}}}\right)$ and the process will be decelerating if $t_{0}+\frac{2}{\gamma \alpha} \ln \left(\frac{\delta+H_{0}}{\delta+\frac{\gamma \alpha}{4}+\sqrt{\frac{\gamma^{2} \alpha^{2}}{16}-\frac{\gamma \mu}{2}}}\right)<t<$ $t_{0}+\frac{2}{\gamma \alpha} \ln \left(\frac{\delta+H_{0}}{\delta+\frac{\gamma \alpha}{4}-\sqrt{\frac{\gamma^{2} \alpha^{2}}{16}-\frac{\gamma \mu}{2}}}\right)$.

We shall now discuss the following particular choices for particle creation rate $\left(\Gamma_{3}=0\right)$ which may be interesting for the present collapse dynamics of a massive star. 


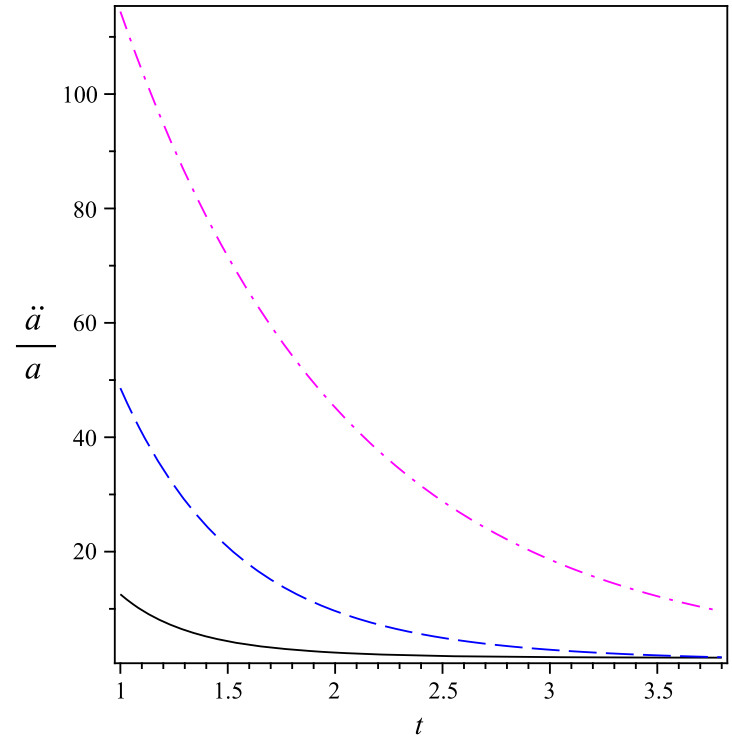

Fig. 2 The figure on the left side represents accelerating collapsing process against time $t$ given by second equation of (15) and the figure on the right side denotes evolution of the Hubble parameter $(H)$, which is given by (14) against $t$, respectively for $\Gamma_{0}=1$. In both the figures,

- $\Gamma=3 \Gamma_{0} H+\frac{\Gamma_{1}}{H}$ : In this case the evolution equation for the scale factor takes the form $\left(\Gamma_{0} \neq 1\right)$

$\frac{\ddot{a}}{a}+\left\{\frac{3 \gamma}{2}\left(1-\Gamma_{0}\right)-1\right\} H^{2}-\frac{\gamma \Gamma_{1}}{2}=0$,

whose solution gives the scale factor and the Hubble parameter as

$$
\begin{aligned}
& a=a_{0}\left[1+\frac{3 \gamma H_{0}}{2}\left(1-\Gamma_{0}\right)\left(t-t_{0}\right)\right]^{\frac{2}{3 \gamma\left(1-\Gamma_{0}\right)}}, \\
& H=\frac{H_{0}}{\left[1+\frac{3 \gamma H_{0}}{2}\left(1-\Gamma_{0}\right)\left(t-t_{0}\right)\right]},
\end{aligned}
$$

for $\Gamma_{0} \neq 1, \Gamma_{1}=0$ and

$$
\begin{aligned}
a= & a_{0}\left[\cosh \left\{\frac{3 \gamma}{2} \sqrt{\Gamma_{2}}\left(1-\Gamma_{0}\right)\left(t-t_{0}\right)\right\}\right. \\
& \left.+\frac{H_{0}}{\sqrt{\Gamma_{2}}} \sinh \left\{\frac{3 \gamma}{2} \sqrt{\Gamma_{2}}\left(1-\Gamma_{0}\right)\left(t-t_{0}\right)\right\}\right]^{\frac{2}{3 \gamma\left(1-\Gamma_{0}\right)}} \\
H= & \sqrt{\Gamma_{2}} \tanh \left[\tanh ^{-1}\left(\frac{H_{0}}{\sqrt{\Gamma_{2}}}\right)+\frac{3 \gamma}{2} \sqrt{\Gamma_{2}}\left(1-\Gamma_{0}\right)\left(t-t_{0}\right)\right],
\end{aligned}
$$

for $\Gamma_{0} \neq 1, \Gamma_{1} \neq 0$ with $\Gamma_{2}=\frac{\Gamma_{1}}{3\left(1-\Gamma_{0}\right)}$. One may note that, the solution (17) can be obtained from the solution (18) in the limiting situation $\Gamma_{1} \rightarrow 0$.

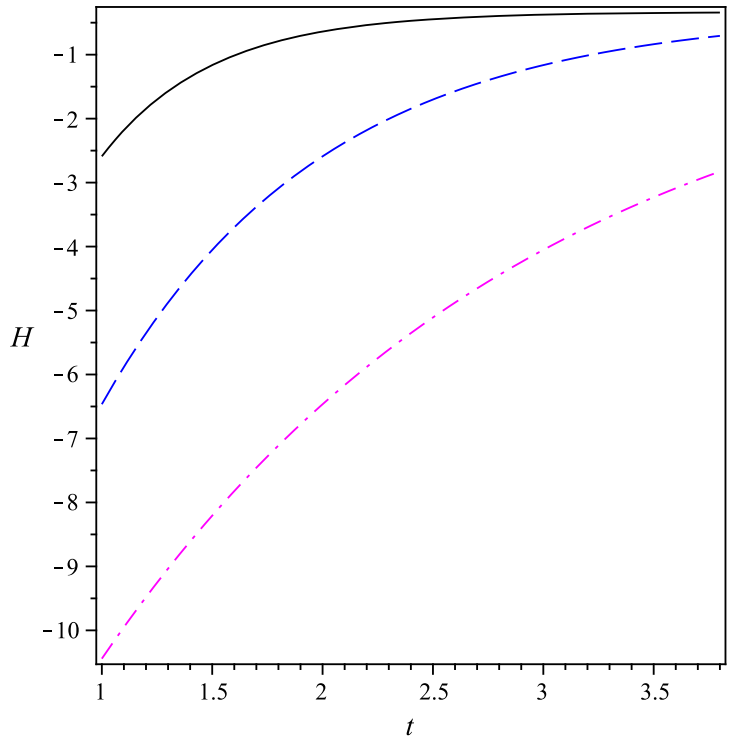

the curves in the solid line represent $\frac{\ddot{a}}{a}$ and $H$, respectively for $\gamma=\frac{4}{3}$. The curves in the dashed line represent $\frac{\ddot{a}}{a}$ and $H$, respectively for $\gamma=\frac{2}{3}$ and the curves in the dash-dotted line represent $\frac{\ddot{a}}{a}$ and $H$, respectively for $\gamma=\frac{1}{3}$. For all the curves we have considered $\alpha=3$ and $\delta=\frac{1}{3}$

The time of collapse $\left(t_{c}\right)$ can be obtained from these solution (by putting $a=0$ ) as

$t_{c}= \begin{cases}t_{0}-\frac{2}{3 \gamma H_{0}\left(1-\Gamma_{0}\right)}, & \text { for } \Gamma_{1}=0 \\ t_{0}-\frac{2}{3 \gamma \sqrt{\Gamma_{2}}\left(1-\Gamma_{0}\right)} \operatorname{coth}^{-1}\left(\frac{H_{0}}{\sqrt{\Gamma_{2}}}\right), & \text { for } \Gamma_{1} \neq 0 .\end{cases}$

The time of formation of apparent horizon (which is characterized by $\left.R H\right|_{t=t_{a H}}=-1$ ) for the present model is given by

$$
\begin{aligned}
t_{a H} & =t_{0}+\frac{2}{3 \gamma H_{0}\left(1-\Gamma_{0}\right)}\left[-1+\left(-\frac{1}{R_{0} H_{0}}\right)^{\frac{1}{l}}\right], \\
l & =\frac{2}{3 \gamma\left(1-\Gamma_{0}\right)}-1, \Gamma_{1}=0,
\end{aligned}
$$

while for $\Gamma_{1} \neq 0, t_{a H}$ is obtained implicitly from the following relation

$$
\begin{aligned}
& R_{0} \sqrt{\Gamma_{2}} \tanh \left[\tanh ^{-1}\left(\frac{H_{0}}{\sqrt{\Gamma_{2}}}\right)+\frac{3 \gamma}{2} \sqrt{\Gamma_{2}}\left(1-\Gamma_{0}\right)\left(t_{a H}-t_{0}\right)\right] \\
& \quad \times\left[\cosh \left\{\frac{3 \gamma}{2} \sqrt{\Gamma_{2}}\left(1-\Gamma_{0}\right)\left(t_{a H}-t_{0}\right)\right\}\right. \\
& \left.\quad+\frac{H_{0}}{\sqrt{\Gamma_{2}}} \sinh \left\{\frac{3 \gamma}{2} \sqrt{\Gamma_{2}}\left(1-\Gamma_{0}\right)\left(t_{a H}-t_{0}\right)\right\}\right]^{\frac{2}{3 \gamma\left(1-\Gamma_{0}\right)}}=-1 .
\end{aligned}
$$

From the above relation it is not possible to obtain an explicit expression for $t_{a H}$. However, in particular, if $\frac{2}{3\left(1-\Gamma_{0}\right)}$ is chosen as unity, then from above relation 


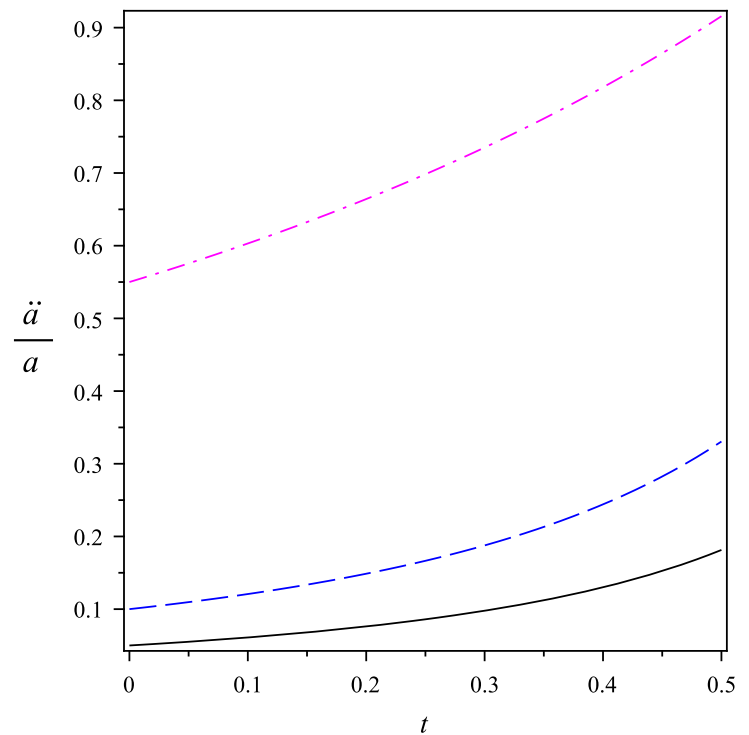

Fig. 3 The figure on the left side depicts accelerating collapsing process [given by the first case of (19)] and the figure on the right side depicts evolution of the Hubble parameter $(H)$ given by (17), respectively against time $t$ for $\Gamma_{1}=0$. The curves in the solid line represent

$t_{a H}=t_{0}+\frac{1}{\sqrt{\Gamma_{2}}} \sinh ^{-1}\left[\frac{\sqrt{\Gamma_{2}} \pm H_{0} \sqrt{1-H_{1}^{2} R_{0}^{2}}}{R_{0} H_{1}^{2}}\right]$,

provided $H_{0}<\sqrt{\Gamma_{2}+\frac{1}{R_{0}^{2}}}$ and we have $H_{1}^{2}=H_{0}^{2}-\Gamma_{2}$. Thus, the time difference between the formation of apparent horizon and the time of collapse (for the choice $\frac{3 \gamma\left(1-\Gamma_{0}\right)}{2}=1$ ) is given by

$t_{a H}-t_{c}=\left\{\begin{array}{cc}\frac{1}{H_{0}}\left(-\frac{1}{R_{0} H_{0}}\right)^{\frac{1}{l}}, & \text { for } \Gamma_{1}=0 \\ \frac{1}{\sqrt{\Gamma_{2}}} \sinh ^{-1}\left[\frac{\sqrt{\Gamma_{2}} \pm H_{0} \sqrt{1-H_{1}^{2} R_{0}^{2}}}{R_{0} H_{1}^{2}}\right] & \\ +\frac{1}{\sqrt{\Gamma_{2}}} \operatorname{coth}^{-1}\left(\frac{H_{0}}{\sqrt{\Gamma_{2}}}\right), & \text { for } \Gamma_{1} \neq 0 .\end{array}\right.$

As $H_{0}$ is negative so $t_{a H}<t_{c}$ for $\Gamma_{1}=0$ i.e. the collapsing star will inevitably lead to $\mathrm{BH}$ in this case. However no definite conclusion can be made about the nature of the collapsing singularity for $\Gamma_{1} \neq 0$. The measure of acceleration for the present choice is given by

$\ddot{a}=\left\{\begin{array}{ll}\frac{\left\{1-\frac{3 \gamma}{2}\left(1-\Gamma_{0}\right)\right\} H_{0}^{2}}{\left[1+\frac{3 \gamma H_{0}}{2}\left(1-\Gamma_{0}\right)\left(t-t_{0}\right)\right]^{2}}, & \text { for } \Gamma_{1}=0 \\ \Gamma_{2}, & \text { for } \Gamma_{1} \neq 0\end{array} \quad\right.$ (with $\left.\frac{3 \gamma}{2}\left(1-\Gamma_{0}\right)=1\right)$.

For $\Gamma_{1}=0$ the collapsing process will be accelerating if $\frac{3 \gamma}{2}\left(1-\Gamma_{0}\right)<1$ and the process will be decelerating otherwise (see Fig. 3), while for $\Gamma_{1} \neq 0$ with $\frac{3 \gamma}{2}\left(1-\Gamma_{0}\right)=1$

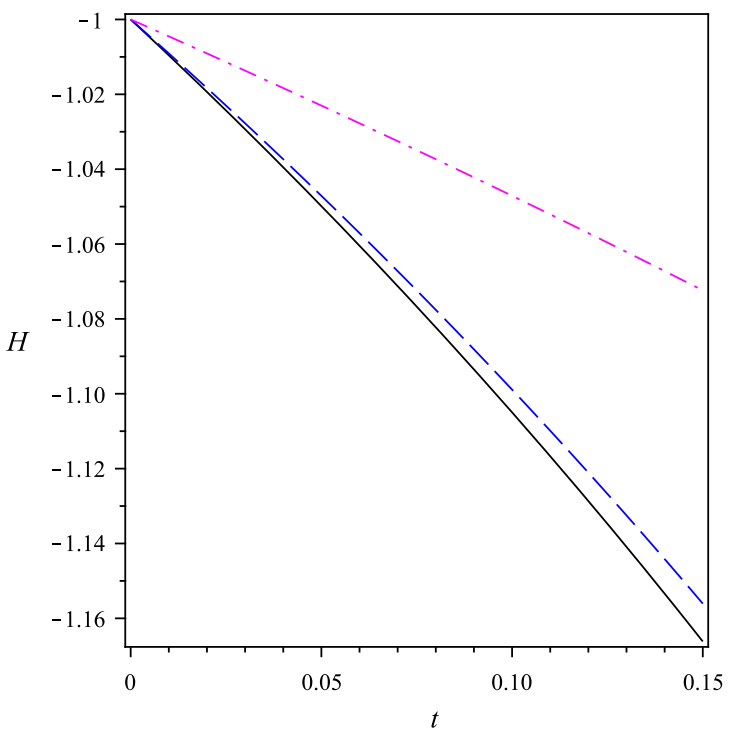

$\frac{\ddot{a}}{a}$ and $H$, respectively for $\Gamma_{0}=-0.9$, the dashed lines represent for $\Gamma_{0}=-0.8$ and the dash-dotted lines represent for $\Gamma_{0}=0.1$, respectively. In all the figures we have considered $\gamma=\frac{1}{3}$

the collapsing process will be accelerating if $\Gamma_{2}>0$ and decelerating if $\Gamma_{2}<0$.

We shall now consider the choice $\Gamma_{0}=1$. For this choice, the evolution Eq. (16) simplifies to

$\dot{H}=\frac{\gamma \Gamma_{1}}{2}$,

which integrating once, we obtain

$H=H_{0}+\frac{\gamma \Gamma_{1}}{2}\left(t-t_{0}\right)$

As collapsing phase of the star is under consideration, so we choose $\Gamma_{1}<0$. Hence the scale factor evolves as

$a=a_{0} \exp \left[H_{0}\left(t-t_{0}\right)+\frac{\gamma \Gamma_{1}}{4}\left(t-t_{0}\right)^{2}\right]$.

It is evident from the above expression of the scale factor that the star requires an infinite time to reach the collapsing singularity (i.e. $t_{c}=\infty$ ). The time of formation of the apparent horizon $\left(t_{a H}\right)$ satisfies the relation

$R_{0}\left[H_{0}+\frac{\gamma \Gamma_{1}}{2} T_{a H}\right] \exp \left[H_{0} T_{a H}+\frac{\gamma \Gamma_{1}}{4} T_{a H}^{2}\right]=-1$,

for which finite solution is possible. Thus, in this case, the star will become a BH in a finite time. From the expression for acceleration i.e.

$\frac{\ddot{a}}{a}=\left\{H_{0}+\frac{\gamma \Gamma_{1}}{2}\left(t-t_{0}\right)\right\}^{2}+\frac{\gamma \Gamma_{1}}{2}$, 


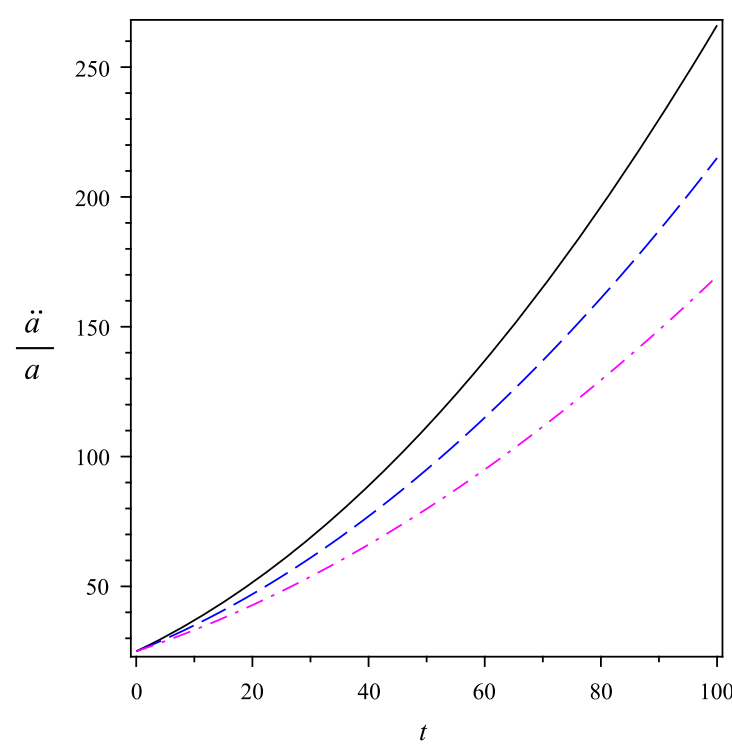

Fig. 4 The figure on the left side shows accelerating collapsing process given by (22) and the figure on the right side shows evolution of Hubble parameter given by (21), respectively against time $t$. In both the figures the curves in the solid line represent $\frac{\ddot{a}}{a}$ for $\Gamma_{1}=-0.7$,

we see that the above collapsing process will be decelerating if $-\sqrt{-\frac{\gamma \Gamma_{1}}{2}}-H_{0}<\frac{\gamma \Gamma}{2}\left(t-t_{0}\right)<\sqrt{-\frac{\gamma \Gamma_{1}}{2}}-H_{0}$, otherwise it is accelerating (see Fig. 4).

- Particular choice of the particle creation parameter $\left(\Gamma_{1}=0\right.$ in (8)) i.e. $\Gamma=3 \Gamma_{0} H+\Gamma_{3}$ : The evolution equation for the scale factor can be obtained from Eq. (9) (by putting $\left.\Gamma_{1}=0\right)$ as

$\frac{\ddot{a}}{a}+\left\{\frac{3 \gamma}{2}\left(1-\Gamma_{0}\right)-1\right\} \frac{\dot{a}^{2}}{a^{2}}-\frac{\gamma \Gamma_{3}}{2} \frac{\dot{a}}{a}=0$.

The relevant physical parameters are given by $\left(\Gamma_{3}>0, \Gamma_{0}=\right.$ 1)

$$
\begin{aligned}
a & =a_{0} \exp \left[\frac{2 H_{0}}{\gamma \Gamma_{3}}\left\{e^{\frac{\gamma \Gamma_{3}}{2}\left(t-t_{0}\right)}-1\right\}\right], \\
H & =H_{0} \exp \left[\frac{\gamma \Gamma_{3}}{2}\left(t-t_{0}\right)\right], \\
t_{c} & =\infty,
\end{aligned}
$$

and $t_{a H}$ is determined by the relation

$$
R_{0} H_{0} e^{\frac{\gamma \Gamma_{3}}{2}\left(t_{a H}-t_{0}\right)} \exp \left[\frac{2 H_{0}}{\gamma \Gamma_{3}}\left\{e^{\frac{\gamma \Gamma_{3}}{2}\left(t_{a H}-t_{0}\right)}-1\right\}\right]=-1 \text {. }
$$

So the collapsing process will inevitably lead to the formation of $\mathrm{BH}$. The acceleration is given by

$$
\frac{\ddot{a}}{a}=H_{0} e^{\frac{\gamma \Gamma 3}{2}\left(t-t_{0}\right)}\left[H_{0} e^{\frac{\gamma \Gamma 3}{2}\left(t-t_{0}\right)}+\frac{\gamma \Gamma_{3}}{2}\right] .
$$

The present collapsing process will be accelerating if $t>t_{0}+$ $\frac{2}{\gamma \Gamma_{3}} \ln \left(-\frac{\gamma \Gamma_{3}}{2} H_{0}\right)$, otherwise it is decelerating (see Fig. 5).

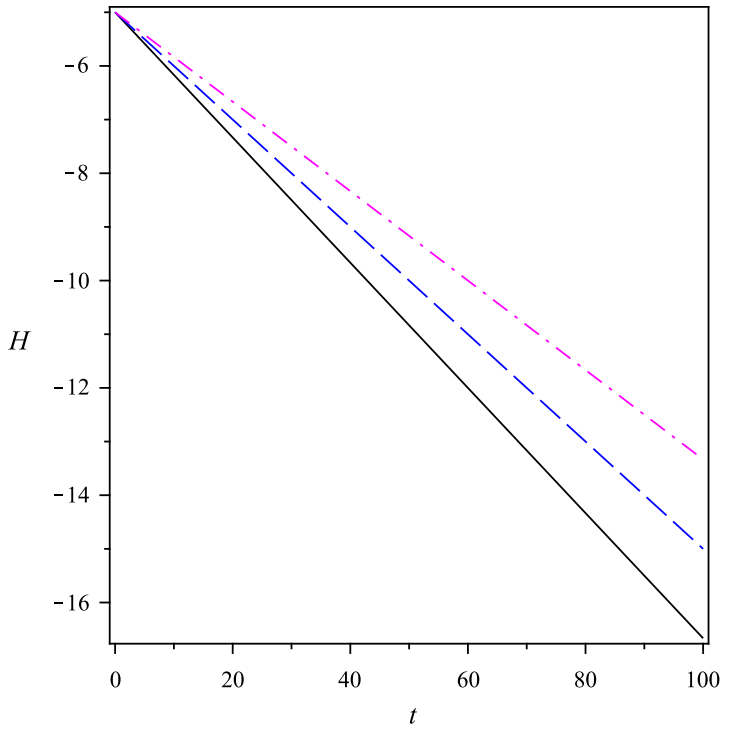

the dashed lines represent for $\Gamma_{1}=-0.6$, and the dash-dotted lines represent for $\Gamma_{1}=-0.5$, respectively. In all the cases here we have considered $\gamma=\frac{1}{3}, t_{0}=0$

However, when $\Gamma_{0} \neq 1$, then the expressions for the above parameters are given by $\left(\Gamma_{3}>0,0<\Gamma_{0}<1\right)$

$$
\begin{aligned}
a & =a_{0}\left[H_{0}\left\{\alpha^{2} e^{\delta\left(t-t_{0}\right)}-\beta^{2}\right\}\right]^{\frac{1}{\alpha^{2} \delta}}, \\
H^{-1} & =\alpha^{2}-\beta^{2} e^{-\delta\left(t-t_{0}\right)}, \\
\ddot{a} & =\frac{\left\{1-\delta \beta^{2} e^{-\delta\left(t-t_{0}\right)}\right\}}{\left\{\alpha^{2}-\beta^{2} e^{-\delta\left(t-t_{0}\right)}\right\}^{2}}, \\
t_{c} & =t_{0}+\frac{1}{\delta} \ln \left(\frac{\beta^{2}}{\alpha^{2}}\right),
\end{aligned}
$$

where $\alpha^{2}=\frac{3\left(1-\Gamma_{0}\right)}{\Gamma_{3}}, \beta^{2}=\alpha^{2}-\frac{1}{H_{0}}, \delta=\frac{\gamma \Gamma_{3}}{2}$ and $t_{a H}$ is determined by the relation

$R_{0}\left(H_{0}\right)^{\left(\frac{1}{\alpha^{2} \delta}\right)} e^{\delta\left(t_{a H}-t_{0}\right)}\left[\alpha^{2} e^{\delta\left(t_{a H}-t_{0}\right)}-\beta^{2}\right]^{\left(\frac{1}{\alpha^{2} \delta}-1\right)}=-1$.

Now using the expression for $t_{c}$ in the determining equation for $t_{a H}$, we have

$\frac{R_{0}}{\alpha^{2}}\left(H_{0} \alpha^{2}\right)^{\left(\frac{1}{\alpha^{2} \delta}\right)} e^{T_{a H}}\left[e^{T_{a H}}-e^{T_{c}}\right]^{\left(\frac{1}{\alpha^{2} \delta}-1\right)}=-1$.

The above equation will be consistent only if $n=\frac{1}{\alpha^{2} \delta}$ is an integer. Further, if $\mathrm{n}$ is an odd integer we always have $T_{a H}>$ $T_{c}$ i.e. the collapse leads to NS. However, if $n$ is an even integer then we always have $T_{a H}<T_{c}$ i.e. the singularity is covered by the apparent horizon, so BH will form. For this case, the collapsing process will be accelerating if $t>t_{0}+$ $\frac{1}{\delta} \ln \left(\delta \beta^{2}\right)$ and decelerating if $t<t_{0}+\frac{1}{\delta} \ln \left(\delta \beta^{2}\right)$ (see Fig. 6). 


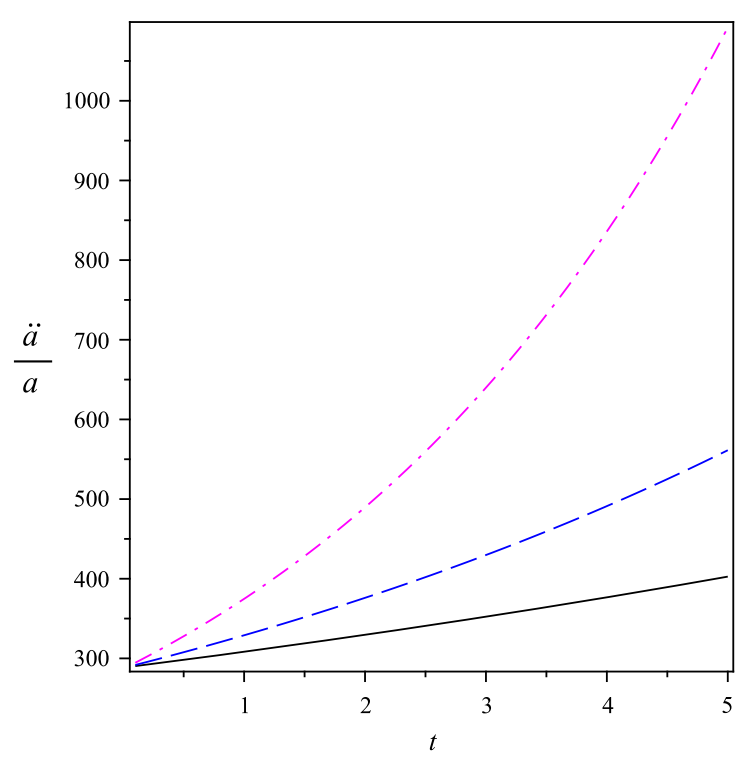

Fig. 5 The figure on the left side shows $\frac{\ddot{a}}{a}$ [given by (25)] vs. time $t$ and the figure on the right side shows evolution of the Hubble parameter given by (24) for $\Gamma=3 H+\Gamma_{3}, \Gamma_{3}>0$. In both the figures, the curves

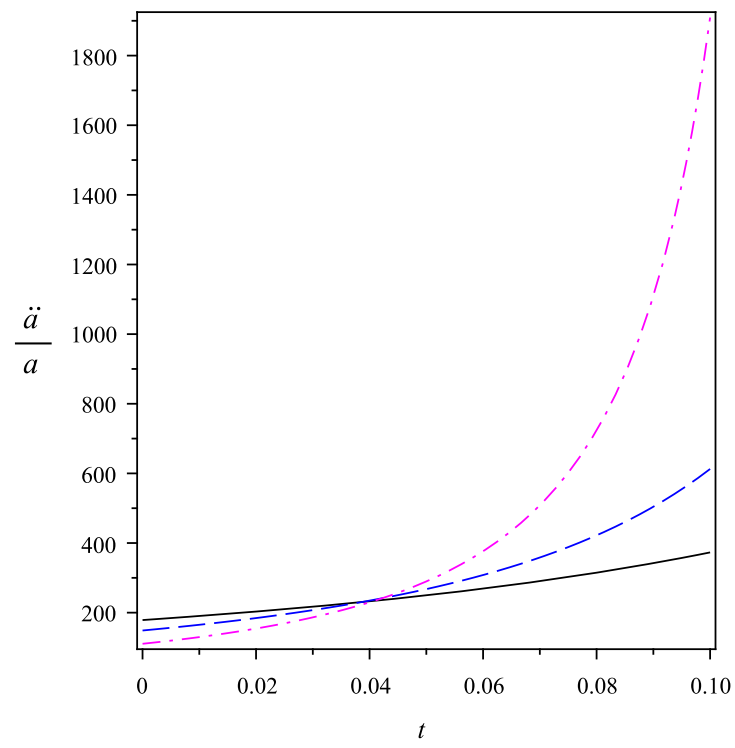

Fig. 6 The figure on the left side shows $\frac{\ddot{a}}{a}$ [given by (27)] vs. time $t$ and the figure on the right side shows evolution of the Hubble parameter given by (26) against time $t$ for $\Gamma=3 \Gamma_{0} H+\Gamma_{3}, \Gamma_{3}>0,0<$

\section{Exterior Schwarzschild-de Sitter spacetime and junction conditions at the surface of the collapsing star}

Let the surface of the collapsing star be $\Sigma$, a time-like $3 D$ hypersurface and it divides the $4 D$ spacetime into two distinct $4 D$ manifolds $M^{ \pm}$. The interior of the collapsing star is the manifold $M^{-}$, described by the flat FRW metric (2), while the exterior manifold $M^{+}$corresponds to Schwarzschild-de

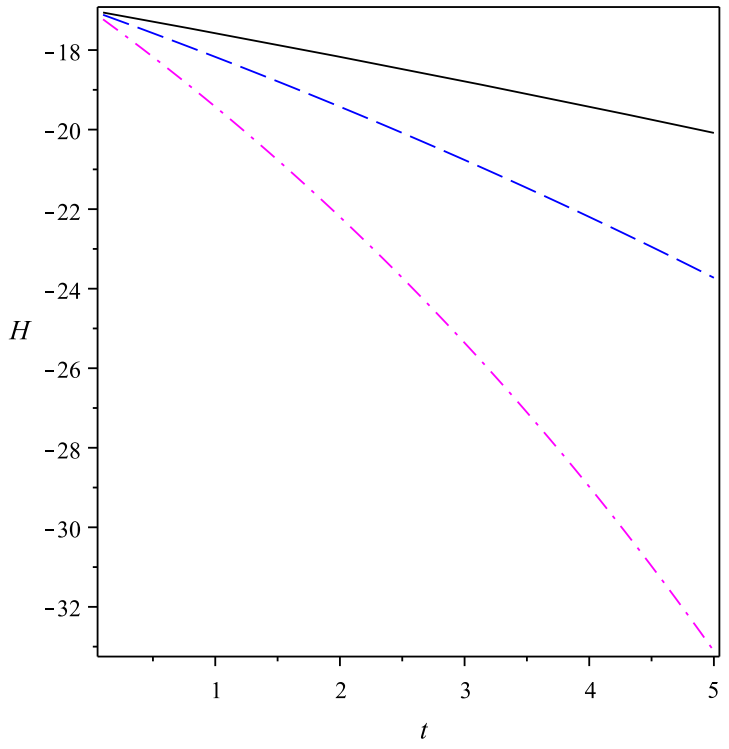

in the solid line represent $\frac{\ddot{a}}{a}$ for $\gamma=\frac{1}{3}$, the dashed lines represent for $\gamma=\frac{2}{3}$ and the dash-dotted lines represent for $\gamma=\frac{4}{3}$, respectively. In all the cases here we have considered $\Gamma_{3}=0.2, t_{0}=0$

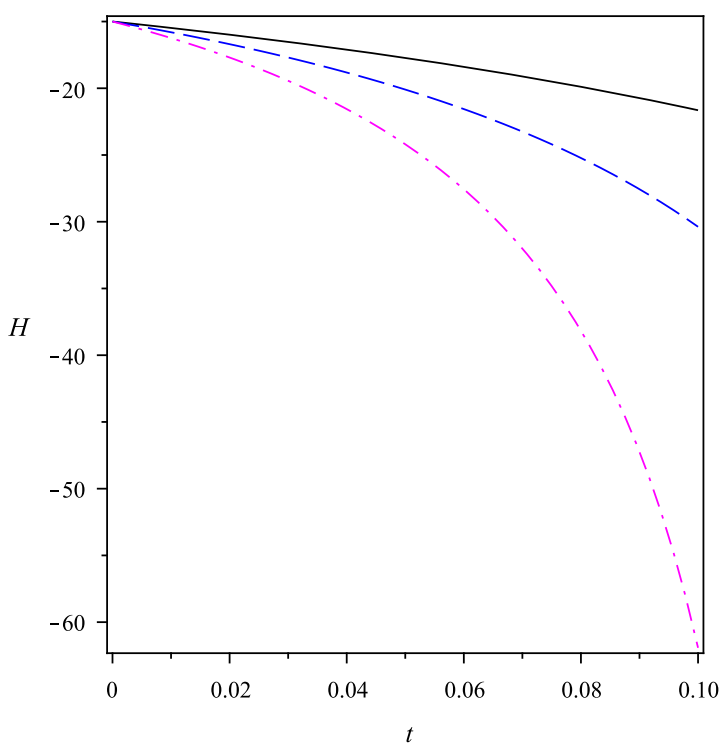

$\Gamma_{0}<1$. In both the figures, the curves in the solid lines represent for $\delta=\frac{1}{12}, \alpha=\frac{2 \sqrt{15}}{5}$, the dashed lines represent for $\delta=\frac{1}{12}, \alpha=2$ and the dash-dotted lines represent for $\delta=\frac{1}{8}, \alpha=2$, respectively

Sitter spacetime having line element

$d s_{+}^{2}=-U(\rho) d T^{2}+\frac{1}{U(\rho)} d \rho^{2}+\rho^{2} d \Omega_{2}^{2}$,

with $U(\rho)=1-\frac{2 M}{\rho}-\left(\frac{\Lambda}{3}\right) \rho^{2}$ where $M$ and $\Lambda$ are constants. Now the intrinsic metric on the surface $\Sigma$ of the collapsing star is chosen as

$d s_{\Sigma}^{2}=-d \tau^{2}+D^{2}(\tau) d \Omega_{2}^{2}$. 
According to Santos [44,45], the Israel's junction [46] conditions across $\Sigma$ are given by

1. Continuity of the line element across the bounding surface $\Sigma$, i.e.

$$
\left(d s_{-}^{2}\right)_{\Sigma}=\left(d s_{+}^{2}\right)_{\Sigma}=d s_{\Sigma}^{2}
$$

where ()$_{\Sigma}$ means the value of the corresponding quantity on the surface $\Sigma$.

2. The extrinsic curvature should be continuous through the bounding surface $\Sigma$, i.e.

$$
K_{a b}^{+}=K_{a b}^{-}, \quad \text { on } \Sigma \text {. }
$$

According to Eisenhart [47], the explicit expression for extrinsic curvature components are

$K_{a b}^{ \pm}=-n_{\mu}^{ \pm} \frac{\partial^{2} \chi_{ \pm}^{\mu}}{\partial \xi^{a} \partial \xi^{b}}-n_{\mu}^{ \pm} \Gamma_{\alpha \beta}^{\mu} \frac{\partial \chi_{ \pm}^{\alpha}}{\partial \xi^{a}} \frac{\partial \chi_{ \pm}^{\beta}}{\partial \xi^{b}}$,

where $\chi_{ \pm}^{\alpha}, \alpha=0,1,2,3$ are the co-ordinates in $M^{ \pm}, n_{\mu}^{ \pm}$are the components of the normal vector to $\Sigma$ in the co-ordinates $\chi_{ \pm}^{\alpha}$, and $\xi^{a}=(\tau, \theta, \phi)$ are the intrinsic co-ordinates to $\Sigma$.

In view of $M^{-}$, the surface $\Sigma$ is described mathematically as

$\Sigma: f_{-}(r, t)=r-r_{\Sigma}=0$,

with $r_{\Sigma}$ as constant. As the vector with components $\frac{\partial f}{\partial \chi_{-}^{\alpha}}$ is orthogonal to $\Sigma$, so the unit normal vector is

$n_{\alpha}^{-}=(0, a, 0,0)$.

Similarly, from the point of view of $M^{+}$, the mathematical description of $\Sigma$ is given by

$\Sigma: f_{+}(\rho, T)=\rho-\rho_{\Sigma}(T)=0$,

with unit normal vector

$n_{\alpha}^{+}=\left[U-\frac{1}{U}\left(\frac{d \rho}{d T}\right)^{2}\right]^{-\frac{1}{2}}\left(-\frac{d \rho}{d T}, 1,0,0\right)$.

Due to the continuity Eq. (29) we have

$\frac{d t}{d \tau}=1, \quad D(\tau)=R_{\Sigma}=r_{\Sigma} a$

and

$\frac{d T}{d \tau}=\left\{U(\rho)-\frac{1}{U(\rho)}\left(\frac{d \rho}{d T}\right)^{2}\right\}^{-\frac{1}{2}}, \quad D(\tau)=\rho_{\Sigma}(T)$.
Thus the coordinate system in the interior and on the boundary are identical [see Eq. (31)] while the exterior time is redshifted compared to the interior or boundary time [see Eq. (32)]. The non-vanishing components of the extrinsic curvature for the interior metric are [using Eq. (30)]

$K_{\tau \tau}^{-}=0, K_{\theta \theta}^{-}=\operatorname{cosec}^{2} \theta K_{\phi \phi}^{-}=r_{\Sigma} a(t)=R_{\Sigma}$,

and those for the exterior spacetime on the boundary $\Sigma$ are [using Eq. (30)]

$K_{\tau \tau}^{+}=-\left[\frac{U \ddot{T}}{\dot{\rho}}+\frac{d U}{d \rho} \dot{T}\right]_{\Sigma}$,

and

$K_{\theta \theta}^{+}=\operatorname{cosec}^{2} \theta K_{\phi \phi}^{+}=[\dot{T} U(\rho) \rho]_{\Sigma}$,

where an overdot denotes differentiation w.r.t. $\tau$.

So from (33) and (35) and using the junction condition (29) together with (32) give the following relations (on $\Sigma$ )

$U=1-\dot{R}^{2}$,

and

$T=\left(1-R^{2} H^{2}\right)^{-1}$.

Using the explicit expression for $U$, we have from Eq. (36),

$\frac{1}{2} \dot{R}^{2}-\frac{M}{R}-\frac{\Lambda}{6} R^{2}=0 \quad$ (on the boundary).

This is nothing but the energy conservation equation on the boundary surface $\Sigma$ [48]. Note that in the absence of the cosmological term $\Lambda$, i.e. if the exterior of the collapsing star is purely Schwarzschild in nature, then from Eq. (38) we see that the Schwarzschild mass $M$ is nothing but the total mass of the collapsing cloud [see Eq. (5)] due to Cahill and McVittie [42]. Further, the presence of the $\Lambda$-term in Eq. (38) shows a repulsive term in the Newtonian potential [49]

$\phi(R)=\frac{M}{R}+\frac{\Lambda}{6} R^{2}$.

\section{Thermodynamics of the collapsing star}

The second law of $\mathrm{BH}$ mechanics states that obeying the energy conditions, the area of the bounding horizon (future outer dynamical horizon in case of collapse) is always nondecreasing. However, in the cosmological context this second law is extended and termed as generalized second law of thermodynamics which states that the sum of the entropy of the 
horizon and the entropy of the matter bounded by the horizon (cosmological horizon) should not decrease with time. However, in case of gravitational collapse entropy of the infalling matter is not considered so one confines himself to the original second law of thermodynamics. For static case entropy is defined as the area of the event horizon while for dynamic case one considers apparent horizon. But apparent horizon is no longer an outer horizon when $\mathrm{BH}$ forms during gravitational collapse and hence second law can not be applicable. This can be resolved by considering trapping horizon of the spacetime as the union of the inner apparent horizon and the outer event horizon i.e. the boundary of the trapping region. Then validity of the second law can be examined both for $\mathrm{BH}$ and naked singularity (in case of collapsing scenario) by seeing the increasing/decreasing nature of the volume of the trapping horizon.

In this section, we shall investigate the validity of the second law of thermodynamics in the present context. For BH thermodynamics, the second law states that the area of a future (outer) horizon is non-decreasing provided the energy conditions are satisfied. However, in the cosmological context, generalized second law of thermodynamics (GSLT) implies the non-decreasing nature of the total entropy (i.e. entropy of the horizon together with the entropy of the matter distribution bounded by the horizon) variation in course of evolution, although there does not have any proper thermodynamic definition of the entropy function considering the microscopic description of spacetime. Fortunately, in case of collapse dynamics, one does not have to take into account of the entropy of the infalling matter, only one has to examine the increasing or decreasing nature of the entropy function at the trapping horizon (i.e. apparent horizon for FRW model). But if the final fate of the collapsing object is a BH then apparent horizon is an inner trapping horizon and second law of BH thermodynamics can not be applied to it [50]. To avoid this difficulty one may redefine the trapping horizon of the spacetime as the union of the inner apparent horizon and the outer event horizon or in other words as the boundary of the trapped region in the spacetime. As irrespective of the final fate of the collapsing object, the volume of the trapped region increases with time, so the entropy of the trapping horizon can be defined as the rate of change of the volume of the trapped region w.r.t. the area radius $R$. Hence when the final fate of a collapsing star is a BH then the entropy of the horizon is defined as [50]

$S_{h}=\pi\left(R_{e h}^{2}-R_{a h}^{2}\right)$,

where $R_{e h}$ is the Schwarzschild radius of the exterior spacetime. On the other hand, in case of NS as the final fate, the horizon entropy is defined as [50]
$S_{h}= \begin{cases}\pi R_{a h}^{2}, & \text { for } t \in\left[t_{c}, t_{a H}\right) \\ \pi R_{e h}^{2}, & \text { for } t \in\left[t_{a H}, \infty\right) .\end{cases}$

Now to examine the validity of the second law of thermodynamics, we consider the two cases namely that either the exterior spacetime is purely Schwarzschild or the Schwarzschildde Sitter model:

a. Schwarzschild spacetime outside the collapsing star:

In this case,

$R_{e h}=2 M=R \dot{R}^{2}$.

So,

$S_{h}=\pi\left(R^{2} \dot{R}^{4}-\frac{1}{H^{2}}\right)=\pi\left(H^{4} R^{6}-\frac{1}{H^{2}}\right)$

when $\mathrm{BH}$ as the end state of collapse and

$S_{h}= \begin{cases}\frac{\pi}{H^{2}}, & \text { for } t \in\left[t_{c}, t_{a H}\right) \\ \pi H^{4} R^{6}, & \text { for } t \in\left[t_{a H}, \infty\right)\end{cases}$

when collapse leads to a NS. Thus

$\dot{S}_{h}= \begin{cases}\pi\left[2\left(2 H^{3} R^{6}+\frac{1}{H^{3}}\right) \dot{H}+6 H^{5} R^{6}\right], & \text { for } t_{a H}<t_{c} \quad \text { (BH) } \\ -\frac{2 \pi}{H^{3}} \dot{H}, & \text { for } t \in\left[t_{c}, t_{a H}\right) \\ 2 \pi H^{3} R^{6}\left[2 \dot{H}+3 H^{2}\right], & \text { for } t \in\left[t_{c}, \infty\right) \text { (NS). }\end{cases}$

In Table 1 we have discussed possible final state, $\dot{S}_{h}$, restrictions for the validity of the 2nd law of thermodynamics and the Hubble parameter for different particle creation rate in the Schwarzschild spacetime outside the collapsing star.

\section{b. Exterior Schwarzschild-de Sitter spacetime.}

Using Eq. (38), the Schwarzschild radius of the exterior spacetime is given by

$R_{e h}=2 M_{s d}=R \dot{R}^{2}-\frac{\Lambda}{3} R^{3}$.

Hence the entropy of the horizon is given by

$$
S_{h}= \begin{cases}\pi\left\{\left(H^{2}-\frac{\Lambda}{3}\right)^{2} R^{6}-\frac{1}{H^{2}}\right\}, & \text { for } t_{a H}<t_{c} \\ \frac{\pi}{H^{2}}, & \text { for } t \in\left[t_{c}, t_{a H}\right) \\ \pi R^{6}\left(H^{2}-\frac{\Lambda}{3}\right)^{2}, & \text { for } t \in\left[t_{a H}, \infty\right) .\end{cases}
$$



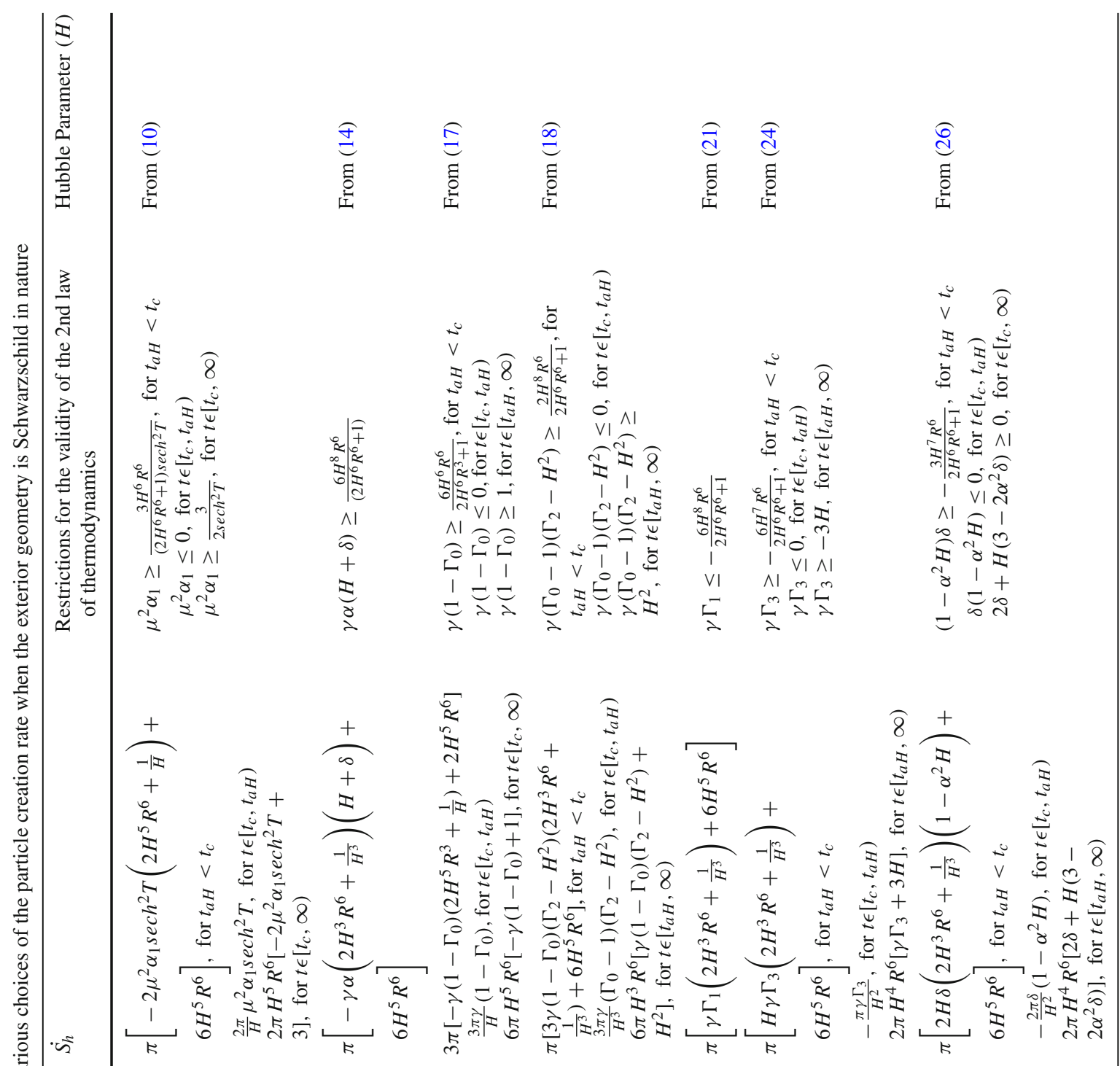
लm
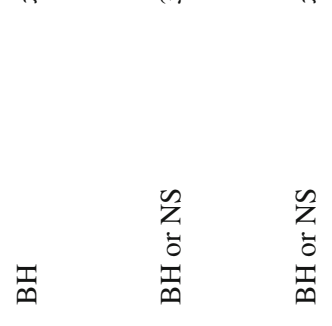

$\stackrel{\square}{\infty}$
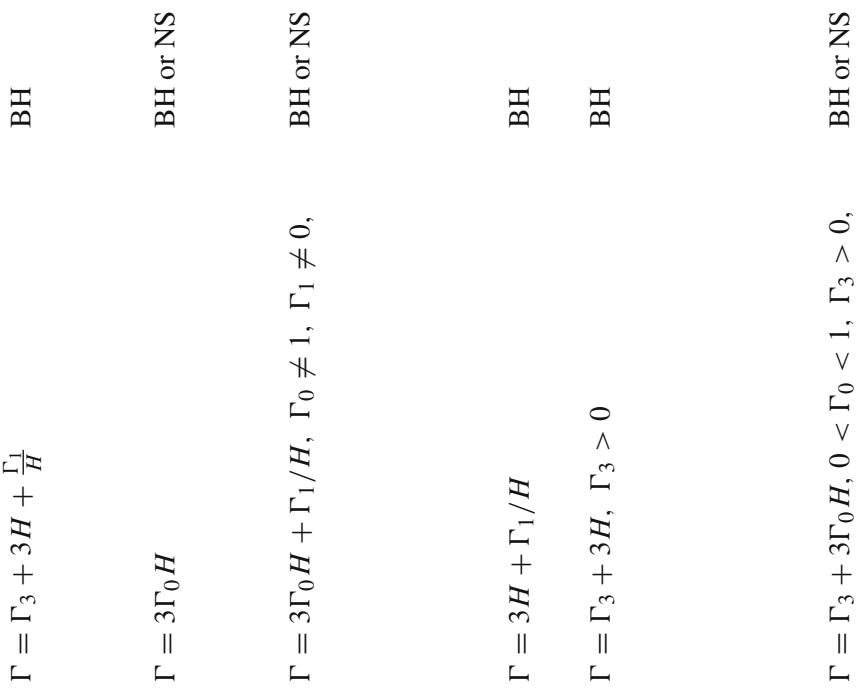
The entropy variation takes the form

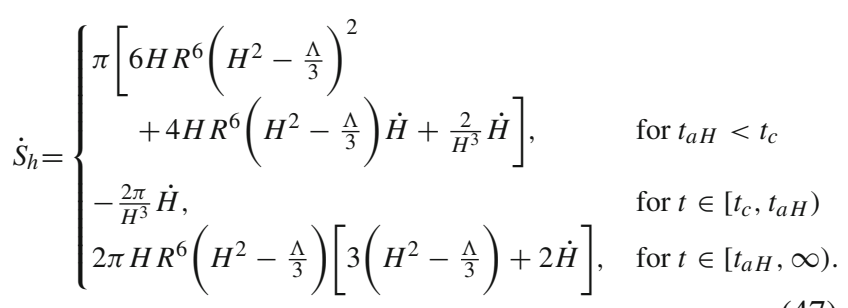

In Table 2 we have discussed possible final state, $\dot{S}_{h}$, restrictions for the validity of the 2 nd law of thermodynamics and the Hubble parameter for different particle creation rate in the Exterior Schwarzschild-de Sitter spacetime.

From both the tables, we see that for the choices second and fifth for the particle creation rate $\mathrm{BH}$ is the definite end state of the collapse, while for the remaining choices we do not have definite conclusion about the final state of collapse - it depends on some restrictions involving the parameters. For thermodynamic analysis, we have defined the horizon entropy [in Eqs. (42), (43) and (46)] separately for the two possible end state of collapse and have examined the validity of the second law of thermodynamics. The tables show that no definite conclusion about the second law of thermodynamics is possible for any form of the particle creation rate parameter (presented in the table), it always involves some inequality related to corresponding parameters.

\section{A field theoretic description of collapsing process}

The section deals with a description of the collapsing process from the field theoretic point of view, i.e. the whole dynamical process (i.e. the collapsing scenario) is considered as the evolution of a scalar field $\phi$ having self interacting potential $V(\phi)$. Equivalently, the collapsing sphere containing effective imperfect fluid can be described by a minimally coupled scalar field. Thus, the energy density and the thermodynamic pressure of the cosmic substrum can be described by the scalar field quantities as

$\rho=\frac{1}{2} \dot{\phi}^{2}+V(\phi) \quad$ and $\quad p_{e f f}=p+\Pi=\frac{1}{2} \dot{\phi}^{2}-V(\phi)$.

Hence for the present adiabatic thermodynamic system scalar field quantities can be expressed as

$\dot{\phi}^{2}=-2 \dot{H} \quad$ and $\quad V(\phi)=3 H^{2}+\dot{H}$.

Note that in the above expression in Eq. (48), we have eliminated the dissipative term $\Pi$ by the adiabatic condition Eq. (1), particle creation rate $\Gamma$ is obtained from the Eq. (8) and we have used the first Friedmann equation (6) to eliminate the energy density $\rho$. Now, from the above expression of (48) both $\phi$ (integrating first equation of (48)) and $V(\phi)$ can be written in parametric form (with $H$ as the parameter) for different particle creation rate as in Table 3.

Although in the table we have presented the potential for the corresponding scalar field as a function of the Hubble parameter but one can easily see that some of the potentials are interesting. For example, for the second and sixth choices the potential may be considered as $\phi^{4}$-type, for the third choice the potential is of exponential type, $\cosh ^{2} \phi$ type potential corresponds to fourth and seventh choices and we have $\phi^{2}$ potential in the fifth choice. One may note that the above canonical scalar field cannot be phantom because in that case $H$ should increase with evolution which is not supported by observation. Moreover, phantom fields lead to instabilities at the classical and quantum levels [56,57]. Further, tachyonic scalar field is not of much interest due to its unstable nature.

\section{Discussion and concluding remarks}

The aim of this paper is to analyze the physical process during collapsing phase of a star. Although we have studied the collapsing scenario due to the local condensation of the perfect fluid, but still we believe that this is true for other matter fields that satisfy the energy conditions. The inside matter of the spherical star is chosen as perfect fluid with barotropic equation state $p=(\gamma-1) \rho \quad(\gamma \neq 0$, a constant $)$. The collapse dynamics is assumed to be a non-equilibrium thermodynamic process having dissipation due to particle creation mechanism. For simplicity, the thermodynamic process is assumed to be adiabatic in nature so that the dissipative effect behaves as bulk viscous pressure and is related linearly to the particle creation rate [see Eq. (1)]. Although the choice of the particle creation rate (8) is purely phenomenological but it has some justification from cosmological scenario [26,29]. Recently, it has been shown that [29] such choice of particle creation rate can describe the cosmic evolution from matter dominated era to present accelerating phase up to phantom barrier. It is generally believed that a collapsing star that consists of homogeneous and isotropic fluid is a very ideal situation, and in more realistic cases the internal region of the collapsing star should be inhomogeneous. However, as argued above, we believe that the main properties of the present model should remain valid even in more realistic situations. Also as expected the collapse dynamics does not depend on the exterior geometry (Schwarzschild/Schwarzschild-de Sitter in the present case).

The curiosity about the collapsing process is due to the lack of definite conclusion about the final fate of the object. It is interesting to examine whether the Cosmic Censorship Conjecture (CCC) of Penrose is obeyed or violated by the collapsing mechanism. We have examined the final fate of 


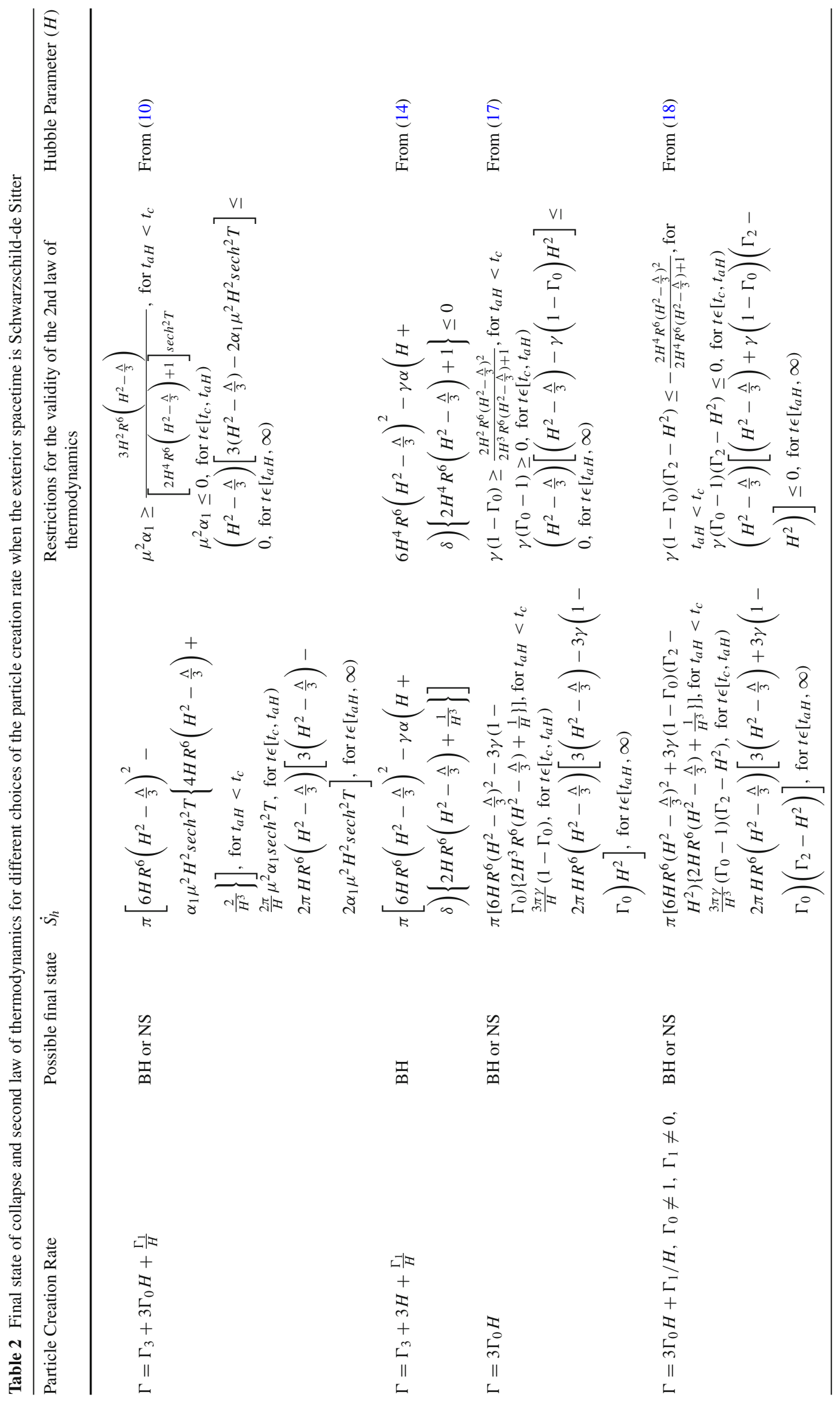




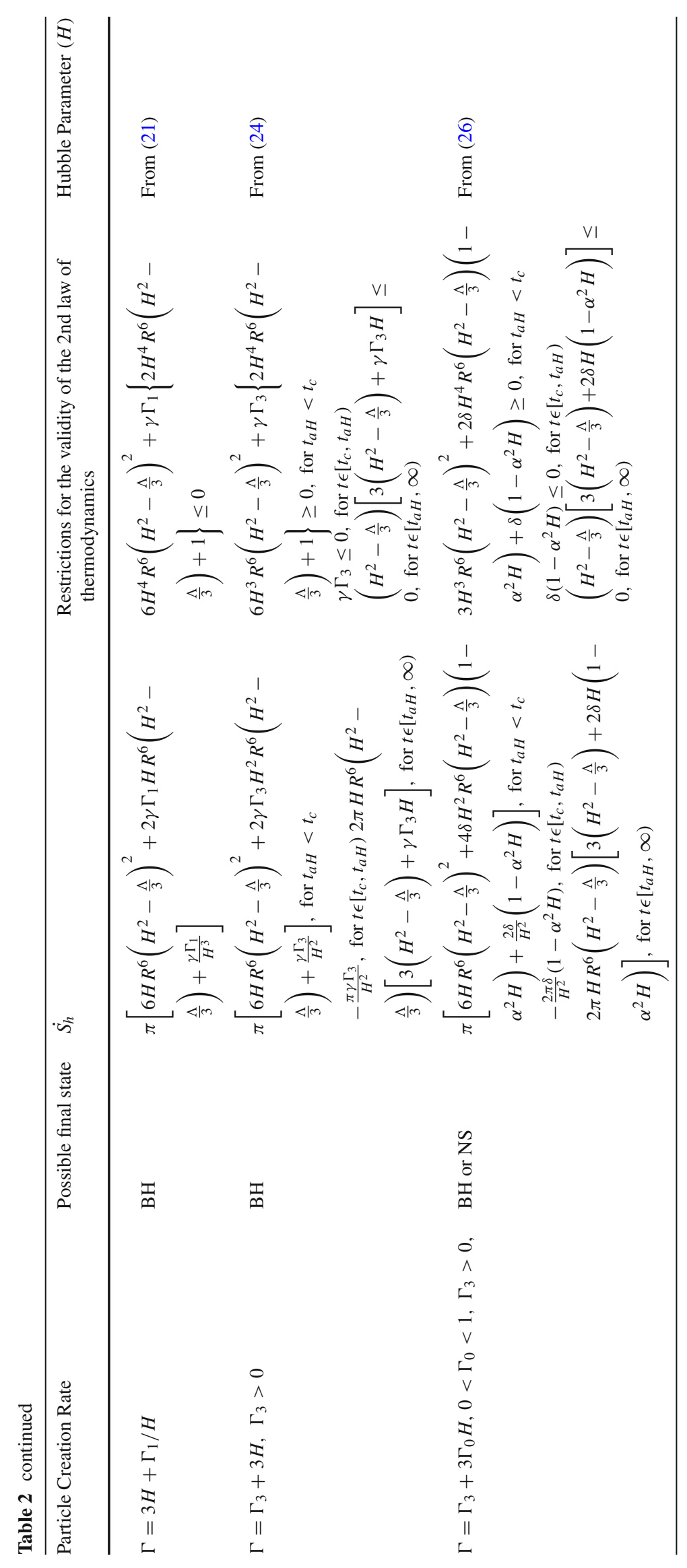




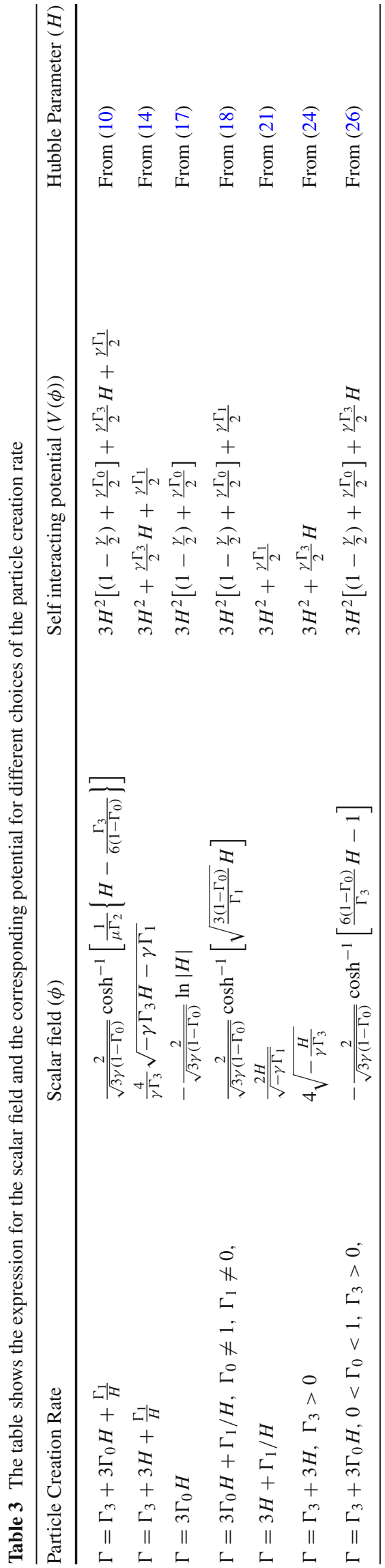

the collapse by comparing the time of collapse and the time of formation of the apparent horizon - whether the final singularity is covered or not by the apparent horizon for various choices of the parameters involved in the choice of the particle creation rate. It is found that in some cases we have definite conclusion about the final phase of collapse (BH or NS) and in other cases depending on some restrictions related to the parameters involved the end state of collapse may be $\mathrm{BH}$ or NS. But in any case formation of $\mathrm{BH}$ is more favoured than NS as the end state of collapse for the present particle creation mechanism.

Also we have examined the thermodynamic laws particularly the second law during the collapsing phase. It is found that depending on some restrictions it is possible to have an increase of entropy during the collapse both for $\mathrm{BH}$ or NS as the final state of collapse. Interestingly, it is found that in some cases where NS is the definite end state the restrictions in the two time intervals namely $\left[t_{c}, t_{a H}\right)$ and $\left[t_{a H}, \infty\right)$ are contradictory. So the second law of thermodynamics will be violated in any one of the time intervals. But in other cases we do not have such definite conclusion.

In the present collapse dynamics of a spherical star, exterior geometry is chosen as Schwarzschild or Schwarzschildde Sitter spacetime. The junction conditions on the boundary show a energy conservation equation on it, the corresponding Newtonian force with cosmological constant [58] is

$F(R)=-\frac{M}{R^{2}}+\frac{\Lambda}{3} R$

As for collapse dynamics, the required force should be attractive in nature, so the area radius $R$ should have an upper bound $\left(\frac{3 M}{\Lambda}\right)^{\frac{1}{3}}$. Also, the rate of collapse is given by

$\ddot{R}=-\frac{M}{R^{2}}+\frac{\Lambda}{3} R$

From the above expression, we may conclude that the collapsing rate is much faster when the exterior spacetime is Schwarzschild rather than the Schwarzschild-de Sitter model. Physically, the cosmological term put some outward pressure to the collapsing star and hence delayed the collapsing process. Also in the context of recent observations the cosmological constant plays the role of dark energy and is best fitted with observations.

Finally, one should note that due to the presence of a cosmological constant (DE), a potential barrier is induced into the equation of motion. As a result, particles with a small velocity will be unable to reach the central object.

Therefore, we conclude that the final fate of a collapsing star in nonequlibrium thermodynamic prescription with particle creation mechanism, depends on the choice of the particle creation rate but in most of the cases the collapsing 
process favours the CCC. For future work, it will be interesting to use this idea astrophysically for a particle orbiting a DE black hole so that an estimation of minimum velocity with which the particle can enter the inside of the $\mathrm{BH}$ and consequently, the amount of $\mathrm{DE}$ inside the $\mathrm{BH}$ may be determined.

Acknowledgements The authors are thankful to the anonymous referee for his/her clarifying comments. SB is thankful to CSIR, Govt. of India for Senior Research Fellowship. SS is partially supported by SERB, Govt. of India under National Post-doctoral Fellowship Scheme [FileNo. PDF/2015/000906]. SC is supported by SERB MATRICS scheme. Also, SC acknowledges UGC-DRS programme at the Department of Mathematics, Jadavpur University. All the authors are thankful to IUCAA, Pune for providing research facilities and warm hospitality while a portion of the work was being done there during a visit.

Open Access This article is distributed under the terms of the Creative Commons Attribution 4.0 International License (http://creativecomm ons.org/licenses/by/4.0/), which permits unrestricted use, distribution, and reproduction in any medium, provided you give appropriate credit to the original author(s) and the source, provide a link to the Creative Commons license, and indicate if changes were made. Funded by SCOAP ${ }^{3}$.

\section{References}

1. S. Perlmutter et al., [Supernova Cosmology Project Collaboration], Discovery of a supernova explosion at half the age of the Universe and its cosmological implications. Nature 391, 51 (1998)

2. S. Perlmutter et al., [Supernova Cosmology Project Collaboration], Measurements of Omega and Lambda from 42 high redshift supernovae. Astrophys. J. 517, 565 (1999)

3. A.G. Riess et al., [Supernova Search Team], Observational evidence from supernovae for an accelerating universe and a cosmological constant. Astron. J. 116, 1009 (1998)

4. A.G. Riess et al., BV RI light curves for 22 type Ia supernovae. Astron. J. 117, 707 (1999)

5. R. Adam et al., [Planck Collaboration], Planck 2015 results. I. Overview of products and scientific results. Astron. Astrophys. 594, A1 (2016)

6. D.J. Eisenstein et al., [SDSS Collaboration], Detection of the Baryon acoustic peak in the large-scale correlation function of SDSS luminous red galaxies. Astrophys. J. 633, 560 (2005)

7. C.R. Contaldi, H. Hoekstra, A. Lewis, Joint CMB and weak lensing analysis: Physically motivated constraints on cosmological parameters. Phys. Rev. Lett. 90, 221303 (2003)

8. S. Boughn, R. Crittenden, A correlation of the cosmic microwave sky with large scale structure. Nature 427, 45 (2004)

9. S. Ray, J.S. Bagla, T. Padmanabhan, Gravitational collapse in an expanding universe: Scaling relations for two-dimensional collapse revisited. Mon. Not. R. Astron. Soc. 360, 546 (2005)

10. L. Parker, Particle creation in expanding universes. Phys. Rev. Lett. 21, 562 (1968)

11. S. Chandrasekhar, The maximum mass of ideal white dwarfs. Astrophys. J. 74, 81 (1931)

12. J.R. Oppenheimer, G.M. Volkoff, On massive neutron cores. Phys. Rev. 55, 374 (1939)

13. S.W. Hawking, G.F.R. Ellis, The Large Scale Structure of SpaceTime (Cambridge University Press, Cambridge, 1973)

14. R. Penrose, Gravitational collapse: The role of general relativity. Riv. Nuovo Cim. 1, 252 (1969)
15. J.R. Oppenheimer, H. Snyder, On continued gravitational contraction. Phys. Rev. 56, 455 (1939)

16. C.W. Misner, D.H. Sharp, Relativistic equations for adiabatic, spherically symmetric gravitational collapse. Phys. Rev. 136, B571 (1964)

17. P. Vaidya, The gravitational field of a radiating star. Proc. Natl. Inst. Sci. India A 33, 264 (1951)

18. A.K.G. de Oliveira, N.O. Santos, Nonadiabatic gravitational collapse. Astrophys. J. 312, 640 (1987)

19. A.K.G. de Oliveira, N.O. Santos, C.A. Kolassis, Collapse of a radiating star. Mon. Not. R. Astron. Soc. 216, 1001 (1985)

20. A.K.G. de Oliveira, J.A. de F. Pacheco, N.O. Santos, More about collapse of a radiating star. Mon. Not. R. Astron. Soc. 220, 405 (1986)

21. A.K.G. de Oliveira, C.A. Kolassis, N.O. Santos, Collapse of a radiating star revisited. Mon. Not. R. Astron. Soc. 231, 1011 (1988)

22. M. Cissoko, J.C. Fabris, J. Gariel, G. Le Denmat, N.O. Santos, Gravitational dust collapse with cosmological constant. arXiv:gr-qc/9809057

23. S.M.C.V. Goncalves, Strong curvature singularities in quasispherical asymptotically de Sitter dust collapse. Class. Quantum Gravity 18, 4517 (2001)

24. D.F. Mota, C. van de Bruck, On the Spherical collapse model in dark energy cosmologies. Astron. Astrophys. 421, 71 (2004)

25. R.G. Cai, A. Wang, Black hole formation from collapsing dust fluid in a background of dark energy. Phys. Rev. D 73, 063005 (2006)

26. S. Chakraborty, S. Saha, A complete cosmic scenario from inflation to late time acceleration: Non-equilibrium thermodynamics in the context of particle creation. Phys. Rev. D. 90(12), 123505 (2014)

27. S. Chakraborty, S. Pan, S. Saha, A third alternative to explain recent observations: Future deceleration. Phys. Lett. B 738, 424 (2014)

28. S. Pan, S. Chakraborty, Will there be future deceleration? A study of particle mechanism in nonequilibrium thermodynamics. Adv. High Energy Phys. 2015, 654025 (2015)

29. S. Chakraborty, S. Pan, S. Saha, A unified cosmic evolution: Inflation to late time acceleration. arxiv:1503.05552 [gr-qc]

30. R.C. Nunes, S. Pan, Cosmological consequences of an adiabatic matter creation process. Mon. Not. R. Astron. Soc. 459(1), 673 (2016)

31. S. Pan, J. de Haro, A. Paliathanasis, R.J. Slagter, Evolution and dynamics of a matter creation model. Mon. Not. R. Astron. Soc. 460(2), 1445 (2016)

32. A. Paliathanasis, J.D. Barrow, S. Pan, Cosmological solutions with gravitational particle production and nonzero curvature. Phys. Rev. D. 95(10), 103516 (2017)

33. W. Zimdahl, Cosmological particle production, causal thermodynamics, and inflationary expansion. Phys. Rev. D 61, 083511 (2000)

34. L. Landau, E.M. Lifschitz, The Classical Theory of Fields (Pergamon Press, New York, 1985)

35. P.J.E. Peebles, Principles of Cosmology (Princeton University Press, Princeton, 1993)

36. S.W. Hawking, Black Holes, ed. by C. de Witt, B.S. de Witt (Gordon and Breach, New York, 1973)

37. P. Anninos, D. Bernstein, S.R. Brandt, D. Hobill, E. Seidel, L. Smarr, Dynamics of black hole apparent horizons. Phys. Rev. D 50, 3801 (1994)

38. U. Debnath, S. Chakraborty, Role of modified Chaplygin gas as a dark energy model in collapsing spherically symmetric cloud. Int. J. Theor. Phys. 47, 2663 (2008)

39. S. Nath, S. Chakraborty, U. Debnath, Gravitational collapse due to dark matter and dark energy in the brane world scenario. Int. J. Mod. Phys. D 15, 1225 (2006)

40. P.S. Joshi, Global aspects in Gravitation and Cosmology (Clarendon, Oxford, 1993) 
41. R.G. Cai, A. Wang, Cosmology with interaction between phantom dark energy and dark matter and the coincidence problem. JCAP 0503, 002 (2005)

42. M.E. Cahill, G.C. McVittie, Spherical symmetry and mass-energy in general relativity. I. General theory. J. Math. Phys. 11, 1382 (1970)

43. D.J. Mortlock et al., A luminous quasar at a redshift of $z=7.085$. Nature 474, 616 (2011)

44. N.O. Santos, Collapse of a radiating viscous fluid. Phys. Lett. A 106, 296 (1984)

45. N.O. Santos, Non-adiabatic radiating collapse. Mon. Not. R. Astron. Soc. 216, 403 (1985)

46. W. Israel, Singular hypersurfaces and thin shells in general relativity. Nuovo Cim. B. 44S10, 1 (1966)

47. L.P. Eisenhart, Riemannian Geometry (Princeton University Press, Princeton, 1949), p. 146

48. U. Debnath, S. Nath, S. Chakraborty, Quasi-spherical collapse with cosmological constant. Mon. Not. R. Astron. Soc. 369, 1961 (2006)

49. A. Balaguera-Antolinez, C.G. Boehmer, M. Nowakowski, Scales set by the cosmological constant. Class. Quantum Gravity 23, 485 (2006)

50. D. Malafarina, P.S. Joshi, Thermodynamics and gravitational collapse. arxiv:1106.3734 [gr-qc]
51. S.A. Hayward, Spin coefficient form of the new laws of black hole dynamics. Class. Quant. Grav. 11, 3025 (1994)

52. S.A. Hayward, Unified first law of black hole dynamics and relativistic thermodynamics. Class. Quantum Gravity 15, 3147 (1998)

53. S.A. Hayward, T. Shiromizu, K.I. Nakao, A cosmological constant limits the size of black holes. Phys. Rev. D 49, 5080 (1994)

54. R.G. Cai, L.M. Cao, Unified first law and the thermodynamics of the apparent horizon in the FRW universe. Phys. Rev. D 75, 064008 (2007)

55. M. Akbar, R.G. Cai, Thermodynamic behavior of Friedmann equations at apparent horizon of FRW universe. Phys. Rev. D 75, 084003 (2007)

56. S.M. Carroll, M. Hoffman, M. Trodden, Can the dark energy equation-of-state parameter w be less than -1? Phys. Rev. D 68, 023509 (2003)

57. J.M. Cline, S. Jeon, G.D. Moore, The Phantom menaced: Constraints on low-energy effective ghosts. Phys. Rev. D 70, 043543 (2004)

58. S. Engineer, N. Kanekar, T. Padmanabhan, Nonlinear density evolution from an improved spherical collapse model. Mon. Not. R. Astron. Soc. 314, 279 (2000) 